\title{
On generalized solutions of two-phase flows for viscous incompressible fluids
}

\author{
Helmut ABels ${ }^{\dagger}$ \\ Max Planck Institute for Mathematics in the Sciences, Inselstr. 22, 04103 Leipzig, Germany
}

[Received 20 December 2005 and in revised form 26 July 2006]

We discuss the existence of generalized solutions of the flow of two immiscible, incompressible, viscous Newtonian and non-Newtonian fluids with and without surface tension in a domain $\Omega \subseteq \mathbb{R}^{d}$, $d=2,3$. In the case without surface tension, the existence of weak solutions is shown, but little is known about the interface between both fluids. If surface tension is present, the energy estimate gives an a priori bound on the $(d-1)$-dimensional Hausdorff measure of the interface, but the existence of weak solutions is open. This might be due to possible oscillation and concentration effects of the interface related to instabilities of the interface as for example fingering, emulsification or just cancellation of area, when two parts of the interface meet. Nevertheless we will show the existence of so-called measure-valued varifold solutions, where the interface is modeled by an oriented general varifold $V(t)$ which is a non-negative measure on $\Omega \times \mathbb{S}^{d-1}$, where $\mathbb{S}^{d-1}$ is the unit sphere in $\mathbb{R}^{d}$. Moreover, it is shown that measure-valued varifold solutions are weak solutions if an energy equality is satisfied.

2000 Mathematics Subject Classification: 35Q30, 35Q35, 76D27, 76D45, 76 T99.

Keywords: Two-phase flow; free boundary problems; varifold solutions; measure-valued solutions; surface tension.

\section{Introduction and main results}

We study the flow of two incompressible, viscous and immiscible fluids like oil and water inside a bounded domain $\Omega$ or in $\Omega=\mathbb{R}^{d}, d=2,3$. The fluids fill domains $\Omega_{+}(t)$ and $\Omega_{-}(t), t>0$, and the interface between both fluids is denoted by $\Gamma(t)$. The flow is described using the velocity $v: \Omega \times(0, \infty) \rightarrow \mathbb{R}^{d}$ and the pressure $p: \Omega \times(0, \infty) \rightarrow \mathbb{R}$ in both fluids in Eulerian coordinates. We assume the fluids to be of a generalized Newtonian type, i.e., the stress tensors are of the form $T^{ \pm}(v, p)=2 v^{ \pm}(|D v|) D v-p I$ with viscosities $v^{ \pm}$depending on the shear rate $|D v|$ of the fluid, $2 D v=\nabla v+\nabla v^{T}$. Moreover, we consider the cases with and without surface tension at the interface. Precise assumptions are made below. Under suitable smoothness assumptions, the flow is a solution of the system

$$
\begin{aligned}
\partial_{t} v+v \cdot \nabla v-\operatorname{div} T^{ \pm}(v, p) & =0 & & \text { in } \Omega^{ \pm}(t), t>0, \\
\operatorname{div} v & =0 & & \text { in } \Omega^{ \pm}(t), t>0, \\
n \cdot T^{+}(v, p)-n \cdot T^{-}(v, p) & =\kappa H n & & \text { on } \Gamma(t), t>0, \\
V & =n \cdot v & & \text { on } \Gamma(t), t>0, \\
v & =0 & & \text { on } \partial \Omega, t>0, \\
\left.v\right|_{t=0} & =v_{0} & & \text { in } \Omega,
\end{aligned}
$$

†E-mail: abels@mis.mpg.de 
together with $\Omega^{+}(0)=\Omega_{0}^{+}$. Here $V$ and $H$ denote the normal velocity and mean curvature, resp., of $\Gamma(t)$ taken with respect to the exterior normal $n$ of $\partial \Omega^{+}(t)$, and $\kappa \geqslant 0$ is the surface tension constant ( $\kappa=0$ means no surface tension is present). Equations (1.1)-(1.2) describe the conservation of linear momentum and mass in both fluids, (1.3) is the balance of forces at the boundary, (1.4) is the kinematic condition that the interface is transported with the flow of the mass particles, and (1.5) is the non-slip condition at the boundary of $\Omega$. Moreover, it is assumed that the velocity field $v$ is continuous along the interface.

Most publications on the mathematical analysis of free boundary value problems for viscous incompressible fluids study quite regular solutions and often deal with well-posedness locally in time or global existence close to equilibrium states (cf. e.g. Solonnikov [29, 30], Beale [3, 4], Tani and Tanaka [33], Shibata and Shimizu [24] or Abels [1]). These approaches are a priori limited to flows in which the interface does not develop singularities and the domain filled by the fluid does not change its topology. In the present contribution we consider certain classes of generalized solutions, which allow singularities of the interface and which exist globally in time for general initial data. For this purpose, we need a suitable weak formulation of the system above. Testing (1.1) with a divergence free vector field $\varphi$ and using in particular the jump relation (1.4), we obtain

$$
-\left(v, \partial_{t} \varphi\right)_{Q}-\left(v_{0},\left.\varphi\right|_{t=0}\right)_{\Omega}-(v \otimes v, \nabla \varphi)_{Q}+(S(\chi, D v), D \varphi)_{Q}=\kappa \int_{0}^{\infty}\left\langle H_{\Gamma(t)}, \varphi(t)\right\rangle \mathrm{d} t
$$

for all $\varphi \in C_{(0)}^{\infty}(\Omega \times[0, \infty))^{d}$ with $\operatorname{div} \varphi=0$, where $Q=\Omega \times(0, \infty), \chi=\chi_{\Omega^{+}}, S(1, D v)=$ $2 v^{+}(|D v|) D v, S(0, D v)=2 v^{-}(|D v|) D v$, and

$$
\left\langle H_{\Gamma(t)}, \varphi(t)\right\rangle=\int_{\Gamma(t)} H n \cdot \varphi(x, t) \mathrm{d} \mathcal{H}^{d-1}(x) .
$$

Now the aim is to construct generalized solutions in a class of functions determined by the energy estimate: If $v$ and $\Gamma(t)$ are sufficiently smooth, then choosing $\varphi=v \chi_{[0, T]}$ in (1.7) one obtains the energy equality

$$
\frac{1}{2}\|v(T)\|_{L^{2}(\Omega)}^{2}+\kappa \mathcal{H}^{d-1}(\Gamma(T))+\int_{0}^{T} \int_{\Omega} S(\chi, D v) D v \mathrm{~d} x \mathrm{~d} t=\frac{1}{2}\left\|v_{0}\right\|_{L^{2}(\Omega)}^{2}+\kappa \mathcal{H}^{d-1}\left(\Gamma_{0}\right)
$$

for all $T>0$, where $\Gamma_{0}=\partial \Omega_{0}^{+}$. Note that $\frac{d}{d t} \mathcal{H}^{d-1}(\Gamma(t))=-\int_{\Gamma(t)} H V \mathrm{~d} \mathcal{H}^{d-1}=-\left\langle H_{\Gamma(t)}, v(t)\right\rangle$ due to (1.4) (cf. Lemma 2.3 below). Now assuming that

$$
v^{ \pm}(|D v|) \geqslant c|D v|^{q-2}
$$

for $q>1$ the equality above gives a uniform bound of

$$
v \in L^{\infty}\left(0, \infty ; L_{\sigma}^{2}(\Omega)\right) \cap L^{q}\left(0, \infty ; \dot{W}_{q}^{1}(\Omega)\right)
$$

where we refer to Section 2.2 below for the precise definitions of the function spaces in this section. Moreover, we note that

$$
-\langle\nabla \chi(t), \varphi\rangle=\int_{\Omega(t)} \operatorname{div} \varphi(x) \mathrm{d} x=\int_{\Gamma(t)} n \cdot \varphi(x) \mathrm{d} \mathcal{H}^{d-1}(x)
$$


for all $\varphi \in C_{0}^{\infty}(\Omega)^{d}$. Hence the distributional gradient $\nabla \chi(t)$ is a finite Radon measure and $\|\nabla \chi(t)\|_{\mathcal{M}(\Omega)}=\mathcal{H}^{d-1}(\Gamma(t))$. Thus, if $\kappa>0$, then $\chi(t) \in B V(\Omega)$ for all $t>0$ and the energy equality above gives an a priori estimate of

$$
\chi \in L^{\infty}(0, \infty ; B V(\Omega)) .
$$

In the case without surface tension, $\kappa=0$, we only find that $\chi \in L^{\infty}(Q)$ is a priori bounded by one. This motivates us to look for weak solutions $(v, \chi)$ lying in the function spaces above, satisfying (1.9) with a suitable substitute of (1.8), such that $(v, \chi)$ solve (1.7) as well as the transport equation

$$
\begin{array}{rlrl}
\partial_{t} \chi+v \cdot \nabla \chi & =0 & & \text { in } Q, \\
\left.\chi\right|_{t=0}=\chi_{0} & & \text { in } \Omega
\end{array}
$$

for $\chi_{0}=\chi_{\Omega_{0}^{+}}$in a suitable weak sense, where 1.10$)$ is a weak formulation of 1.4 (cf. [17, Lemma $1.2])$.

In the case without surface tension and for Newtonian fluids, i.e., $v^{ \pm}(|D v|) \equiv v^{ \pm}>0$, the existence of weak solutions (even for $N$-fluids with different densities) was proven by Nouri and Poupaud [17]. Moreover, Giga and Takahashi [10] consider the case of a two-phase Stokes flow with $v^{+}$close to $v^{-}$. The main difference in their approach is that $1.10-(1.11$ is replaced by a transport equation for a level set function, which is solved in the sense of viscosity solutions. Due to a lack of regularity in the velocity $v$ only sub- and supersolutions exists, which may differ. This causes the possibility of "boundary fattening" (cf. [10] for details). In [17] and the present contribution the transport equation is solved in the sense of renormalized solutions due to DiPerna and Lions [9]. But also the result of Nouri and Poupaud does not give good information for the interface $\Gamma(t)$ since $\Omega^{+}(t)=\{x \in \Omega: \chi(t)=1\}$ is only known to be a measurable set. Moreover, we note that Wagner [35] considered generalized solutions of a one-phase flow for an ideal, irrotational and incompressible fluid and that Gomez and Zolésio [11] treated a quasi-stationary two-phase flow for shear thinning fluids.

Because of the better a priori estimate in the case with surface tension, one might expect to get better results in this case. But unfortunately the additional mean curvature term causes severe problems in the construction of weak solution, which might be related to instabilities of the boundary when fingering or emulsification takes place (cf. e.g. Joseph and Renardy [12]). The only known results for generalized solutions in the case of surface tension are due to Plotnikov [20] for a two-dimensional flow of shear thickening fluids (i.e. $q>d=2$ above) and [21] for the case of compressible fluids, as well as Salvi [23] for an incompressible viscous Newtonian fluid. In Plotnikov's contributions the mean curvature term is interpreted as the first variation of a so called general varifold and it is shown that for almost all $t>0$ the varifold is supported on a rectifiable closed curve dividing the plane into two disjoint domains $\Omega^{ \pm}(t)$. The latter solutions can be considered as some kind of measure-valued solutions and are related to the solutions constructed in the present contribution. In [23] no interpretation of the mean curvature term for the constructed weak solution is given.

It is the purpose of this article to introduce a notion of so called measure-valued varifold solutions of the two-phase flow described above. The definitions are in the spirit of measure-valued solutions for conservation laws and the flow of non-Newtonian fluids as studied for example in [16]. Measure-valued solutions were introduced in order to model possible oscillation and concentration effects on an infinitesimal scale, which mathematically do not allow proving the convergence of 
a suitable approximation scheme to a weak solution. In the present two-phase flow we have to deal with possible oscillation/concentration effects of the shear tensor $D v(x, t)$ as well as of the boundary $\Gamma(t)$. Therefore the definition of a measure-valued varifold solution uses the Young measure generated by the shear tensors $D v_{\varepsilon}(x, t)$ of an approximate sequence $\left(v_{\varepsilon}, \chi_{\varepsilon}\right), \varepsilon>0$, as well as an oriented general $(d-1)$-varifold $V(t)$ generated by the sequence of surfaces $\Gamma_{\varepsilon}(t)$ of the approximation. Here a generalized $(d-1)$-varifold $V$ is simply a non-negative measure $V \in \mathcal{M}\left(\Omega \times \mathbb{S}^{d-1}\right)$, which by disintegration can be represented as a non-negative measure $|V| \in \mathcal{M}(\Omega)$, corresponding to a surface measure, together with a family of probability measures $V_{x}, x \in \Omega$, for the normal vector of the "surface" $n \in \mathbb{S}^{d-1}$, which models possible infinitesimal oscillations of the interface.

Before we come to the precise definitions and results we make the following assumptions:

ASSUMPTION 1.1 We assume that $\kappa>0$ and $\Omega=\mathbb{R}^{d}$ or that $\kappa=0$ and $\Omega \subseteq \mathbb{R}^{d}$ is a bounded domain with Lipschitz boundary or $\Omega=\mathbb{R}^{d}, d=2,3$. Moreover, let $q>1$ and let $v(j, s), j=0,1$, be twice continuously differentiable for $s>0$ such that $v(j, s) s^{2}$ is continuous at 0 and

$$
c_{0} s^{q-2} \leqslant v(j, s) \leqslant C_{0} s^{q-2}, \quad \frac{\mathrm{d}}{\mathrm{d} s}(v(j, s) s)>0, \quad \frac{\mathrm{d}^{2}}{\mathrm{~d} s^{2}}\left(v(j, s) s^{2}\right)>0
$$

for some constants $c_{0}, C_{0}>0$. Finally, we set $S(\theta, A)=\theta v(1,|A|) A+(1-\theta) v(0,|A|) A$ for every $A \in \mathbb{R}_{\mathrm{sym}}^{d \times d}, \theta \in[0,1]$, and $V_{q}(\Omega)=W_{q, 0}^{1}(\Omega)^{d} \cap L_{\sigma}^{q}(\Omega)$ if $\Omega$ is a bounded domain and $V_{q}\left(\mathbb{R}^{d}\right)=\left\{v \in \dot{W}_{q}^{1}\left(\mathbb{R}^{d}\right)^{d}: \operatorname{div} v=0\right\}$.

We note that the simple power law $v(j, s)=v_{j} s^{q-2}$ satisfies the conditions above.

Before defining generalized solutions of the two-phase flow with surface tension we need some notation: An (oriented) general varifold is a non-negative $V \in \mathcal{M}\left(\mathbb{R}^{d} \times \mathbb{S}^{d-1}\right)$. For such a general varifold $V$,

$$
\langle\delta V, \varphi\rangle=\int_{\mathbb{R}^{d} \times \mathbb{S}^{d-1}}(I-s \otimes s): \nabla \varphi(x) \mathrm{d} V(x, s), \quad \varphi \in C_{0}^{1}\left(\mathbb{R}^{d}\right),
$$

denotes its first variation. Moreover, let $Q:=\Omega \times(0, \infty), Q_{t}=\Omega \times(0, t)$, and let $(\cdot, \cdot)_{M}$ denote the $L^{2}$-scalar product on $M$.

Definition 1.2 Let $\kappa>0$ and let Assumption 1.1 hold. Then $v \in L^{\infty}\left(0, \infty ; L_{\sigma}^{2}\left(\mathbb{R}^{d}\right)\right) \cap$ $L^{q}\left(0, \infty ; V_{q}\left(\mathbb{R}^{d}\right)\right), \chi \in L^{\infty}\left(0, \infty ; B V\left(\mathbb{R}^{d} ;\{0,1\}\right)\right), \mu \in L_{\omega}^{\infty}\left(Q ; \operatorname{Prob}\left(\mathbb{R}_{\mathrm{sym}}^{d \times d}\right)\right)$, and $V \in$ $L_{\omega}^{\infty}\left(0, \infty ; \mathcal{M}\left(\mathbb{R}^{d} \times \mathbb{S}^{d-1}\right)\right), V(t) \geqslant 0$ for a.e. $t>0$, is called a measure-valued varifold solution of the two-phase flow for initial data $v_{0} \in L_{\sigma}^{2}\left(\mathbb{R}^{d}\right), \chi_{0}=\chi_{\Omega_{0}^{+}}$for a bounded domain $\Omega_{0}^{+} \Subset \mathbb{R}^{d}$ of finite perimeter if

$$
\begin{aligned}
-\left(v, \partial_{t} \varphi\right)_{Q}-\left(v_{0}, \varphi(0)\right)_{\mathbb{R}^{d}}-(v \otimes v, \nabla \varphi)_{Q} & \\
& +\left(\int_{\mathbb{R}_{\text {sym }}^{d \times d}} S(\chi, \lambda) \mathrm{d} \mu_{x, t}(\lambda), D \varphi\right)_{Q}=-\kappa \int_{0}^{T}\langle\delta V(t), \varphi(t)\rangle \mathrm{d} t
\end{aligned}
$$

for all $\varphi \in C_{(0)}^{\infty}\left(\mathbb{R}^{d} \times[0, \infty)\right)^{d}$ with $\operatorname{div} \varphi=0$, and

$$
\int_{\mathbb{R}^{d} \times \mathbb{S}^{d-1}} s \cdot \psi(x) \mathrm{d} V(t)(x, s)=-\int_{\mathbb{R}^{d}} \psi \mathrm{d} \nabla \chi(t), \quad \psi \in C_{0}\left(\mathbb{R}^{d}\right)^{d},
$$




$$
\int_{\mathbb{R}_{\mathrm{sym}}^{d \times d}} \lambda \mathrm{d} \mu_{x, t}(\lambda)=D v(x, t)
$$

for almost all $(x, t) \in Q, \chi$ is the unique renormalized solution of the transport equation (1.10)1.11) (cf. Section 2.5 below), and $(v, \chi, V, \mu)$ satisfies the generalized energy inequality

$$
\frac{1}{2}\|v(t)\|_{2}^{2}+\kappa\|V(t)\|_{\mathcal{M}}+\int_{Q_{t}} \int S(\chi, \lambda): \lambda \mathrm{d} \mu_{x, \tau} \mathrm{d}(x, \tau) \leqslant \frac{1}{2}\left\|v_{0}\right\|_{2}^{2}+\kappa\left\|\nabla \chi_{0}\right\|_{\mathcal{M}}
$$

for almost all $t \in(0, \infty)$.

REMARK 1.3 1. If $V(t)$ is obtained from a $C^{1}$-surface $\Gamma(t)$ in the natural manner, $\langle\delta V(t), \cdot\rangle$ coincides with the first variation of $\mathcal{H}^{d-1}\lfloor\Gamma(t)$ (cf. Section 2.3 below).

2. Note that by the assumption on $v(j, s), \lambda \mapsto S(\chi, \lambda): \lambda, \lambda \in \mathbb{R}_{\mathrm{sym}}^{d \times d}$, is a strictly convex function. Therefore by the generalized Jensen inequality (cf. 2.4) below) and (1.16),

$$
\int_{Q_{t}} S(\chi, D v): D v \mathrm{~d}(x, \tau) \leqslant \int_{Q_{t}} \int_{\mathbb{R}_{\text {sym }}^{d \times d}} S(\chi, \lambda): \lambda \mathrm{d} \mu_{x, \tau} \mathrm{d}(x, \tau)
$$

for almost all $(x, \tau) \in Q_{t}$, with equality if and only if $\mu_{x, \tau}=\delta_{D v(x, \tau)}$.

3. Let $\left(V_{x}(t),|V(t)|\right), x \in \mathbb{R}^{d}$, denote the disintegration of $V(t) \in \mathcal{M}\left(\mathbb{R}^{d} \times \mathbb{S}^{d-1}\right)$ into a nonnegative measure $|V(t)|$ and a family of probability measures $V_{x}(t) \in \mathcal{M}\left(\mathbb{S}^{d-1}\right)$ as described in Section 2.3 below. Then 1.15 implies that $|\nabla \chi(t)|(A) \leqslant|V(t)|(A)$ for all open sets $A$ and almost all $t \in(0, \infty)$ (cf. $(2.2)$ below). Hence $|\nabla \chi(t)|$ is absolutely continuous with respect to $|V(t)|$ and

$$
\int_{\mathbb{R}^{d}} f(x) \mathrm{d}|\nabla \chi(t)|=\int_{\mathbb{R}^{d}} f(x) \theta_{t}(x) \mathrm{d}|V(t)|, \quad f \in C_{0}\left(\mathbb{R}^{d}\right),
$$

for a $|V(t)|$-measurable function $\theta_{t}: \mathbb{R}^{d} \rightarrow[0, \infty)$ with $\left|\theta_{t}(x)\right| \leqslant 1$ almost everywhere. In particular, this implies supp $\nabla \chi_{t} \subseteq \operatorname{supp} V(t)$ and $\|\nabla \chi(t)\|_{\mathcal{M}} \leqslant\|V(t)\|_{\mathcal{M}}$ for almost all $t \in$ $(0, \infty)$. Hence every measure-valued varifold solution satisfies the energy inequality

$$
\frac{1}{2}\|v(t)\|_{2}^{2}+\kappa\|\nabla \chi(t)\|_{\mathcal{M}}+(S(\chi, D v), D v)_{\Omega_{t}} \leqslant \frac{1}{2}\left\|v_{0}\right\|_{2}^{2}+\kappa\left\|\nabla \chi_{0}\right\|_{\mathcal{M}}
$$

for almost all $t>0$. Moreover, if $E(t)=\left\{x \in \mathbb{R}^{d}: \chi(x, t)=1\right\}, t>0$, then $E(t)$ is for almost every $t>0$ a set of finite perimeter (cf. Section 2.4 below), and (1.15) yields the relation

$$
\int_{\mathbb{S}^{d-1}} s \mathrm{~d} V_{x}(t)(s)= \begin{cases}\theta_{t}(x) n(x) & \text { if } x \in \partial^{*} E_{t}, \\ 0 & \text { else }\end{cases}
$$

for $|V(t)|$-almost every $x \in \mathbb{R}^{d}$ and almost every $t>0$, where $n=-\nabla \chi(t) /|\nabla \chi(t)|$ is the exterior normal of the reduced boundary $\partial^{*} E_{t}$ of $E_{t}$ and $\chi(t)=\chi_{E_{t}}$. In other words, the expectation of $V_{x}(t)$ is proportional to the normal $n$ on the interface and zero inside the fluid.

4. In general, it is an open problem whether $V(t)$ is a so-called countably $(d-1)$-rectifiable varifold, which implies that $V_{x}(t)$ is a Dirac measure for $|V(t)|$-almost every $x$. Then $V(t)$ can naturally be identified with a countably $(d-1)$-rectifiable set—a "surface"—equipped with a density $\theta_{t} \geqslant 0$. So far we can only give a sufficient condition for the rectifiability of $V(t)$ in terms of a regularity condition for the pressure $p(t)$ or the first variation $\delta V(t)$. See Appendix A below for details. 
An open question is whether there are measure-valued varifold solutions such that the first variation $\langle\delta V, \cdot\rangle$ coincides with the negative mean curvature functional associated to $\chi(t)$, which is defined below, and such that $\mu_{x, t}$ coincides with the Dirac measure $\delta_{D v(x, t)}$ almost everywhere. If this is the case, we call $(v, \chi)$ a weak solution:

DEFINITION 1.4 Let $(v, \chi, V, \mu)$ be a measure-valued varifold solution of the two-phase flow in the sense of Definition 1.2. Then $(v, \chi, V)$ is called a varifold solution if $\mu_{x, t}=\delta_{D v(x, t)}$ for almost all $(x, t) \in Q$. If $(v, \chi, V)$ is a varifold solution, then $(v, \chi)$ is called a weak solution of the two-phase flow if

$$
\langle\delta V(t), \psi\rangle=-\left\langle H_{\chi(t)}, \psi\right\rangle=\int_{\mathbb{R}^{d}} \operatorname{Tr}\left(P_{\tau} \nabla \psi\right) \mathrm{d}|\nabla \chi(t)| \quad \text { for all } \psi \in C_{0, \sigma}^{\infty}\left(\mathbb{R}^{d}\right)
$$

and almost all $t \in(0, \infty)$, where $P_{\tau}=I-\frac{\nabla \chi(t)}{|\nabla \chi(t)|} \otimes \frac{\nabla \chi(t)}{|\nabla \chi(t)|}$ (cf. 2.5 below).

From the definitions one derives the following general properties of measure-valued varifold solutions:

Proposition 1.5 (Properties of measure-valued varifold solutions) Let $(v, \chi, V, \mu)$ be a measure-valued varifold solution. Then:

1. If $(v, \chi, V)$ satisfies the energy equality

$$
\frac{1}{2}\|v(t)\|_{2}^{2}+\kappa\|\nabla \chi(t)\|_{\mathcal{M}}+(S(\chi, D v), D v)_{Q_{t}}=\frac{1}{2}\left\|v_{0}\right\|_{2}^{2}+\kappa\left\|\nabla \chi_{0}\right\|_{\mathcal{M}}
$$

for almost all $t \in(0, \infty)$, then $(v, \chi)$ is a weak solution. Moreover, if 1.20 holds with $\|\nabla \chi(t)\|_{\mathcal{M}}$ replaced by $\|V(t)\|_{\mathcal{M}}$, then $(v, \chi, V)$ is a varifold solution.

2. If $q>d$, then $\chi \in B V\left(Q_{T}\right)$ for every $0<T<\infty$.

Our main result concerns existence of measure-valued varifold solutions with some additional properties:

THEOREM 1.6 (Existence of measure-valued varifold solutions) Let $q>2 d /(d+2)$, let $v_{0} \in$ $L_{\sigma}^{2}\left(\mathbb{R}^{d}\right)$, let $\Omega_{0}^{+} \Subset \mathbb{R}^{d}$ be a bounded $C^{1}$-domain, and let $\chi_{0}:=\chi_{\Omega_{0}^{+}}$. Then there is a measurevalued varifold solution $(v, \chi, V, \mu)$ of the two-phase flow as in Definition 1.2. Moreover,

1. If $d=2$ or $q>d$, then $\operatorname{supp} V(t) \subseteq \overline{B_{R}(0)}$ for all $t \in[0, T]$ for some $R=R\left(T, \chi_{0}, v_{0}\right)$ and arbitrary $T>0$.

2. If $q>d=2$, then $(v, \chi, V)$ is a varifold solution and supp $|V(t)|=\Gamma_{t}^{*}$ is a compact rectifiable set and $|V(t)| \geqslant \mathcal{H}^{1}\left\lfloor\Gamma_{t}^{*}\right.$ for almost all $t>0$. Moreover,

$$
d_{H}\left(\Gamma_{t_{1}}^{*}, \Gamma_{t_{2}}^{*}\right) \leqslant C\left|t_{1}-t_{2}\right|^{1 / q^{\prime}} \quad \text { for all } 0 \leqslant t_{1}, t_{2}<\infty,
$$

where $d_{H}(\cdot, \cdot)$ denotes the Hausdorff distance.

REMARK 1.7 The case $q>d=2$ was already studied by Plotnikov in [20], where a similar result is shown, but his definition of a varifold solution is different: Properties of $V(t)$ and $\operatorname{supp}|V(t)|=$ $\Gamma_{t}^{*}$, which can be shown for $q>d=2$, are taken as part of the definition of a varifold solution. In particular, it is required that $\operatorname{supp} V(t)$ is a compact 1-rectifiable set separating the plane into two open sets $\omega_{0}(t)$ and $\omega_{1}(t)$. Moreover, the relation 1.15 is not used. Instead $\chi(t)$ is taken as the characteristic function of the set $\omega_{0}(t)$ and it solves the transport equation in the weak sense. Furthermore, it is required that the space-time interface $\bigcup_{t \in[0, T]} \Gamma_{t}^{*} \times\{t\}$ has for almost every 
$t \in[0, T]$ and every $x \in \Gamma_{t}^{*}$ a tangent plane containing $(v, 1)$. Finally, no energy estimate is part of the definition. See [20] for details.

REMARK 1.8 We note that in the case of a Newtonian fluid, i.e. $v(j,|D v|) \equiv v_{j}$, the proof of Theorem 1.6 yields a conditional existence result for weak solutions if there is no loss of area when passing to the limit in the approximation scheme, i.e. $\lim _{k \rightarrow \infty}\left\|\nabla \chi_{k}(t)\right\|=\|\nabla \chi(t)\|$ for almost all $t>0$. Then the arguments in the proof of Proposition 1.5 or a convergence theorem by Reshetnyak [2, Theorem 2.39] show that $(v, \chi)$ is a weak solution. Such results are known for example for the mean curvature flow by Luckhaus and Sturzenhecker [15] and for the multi-phase Mullins-Sekerka problem by Bronsard, Garcke, and Stoth [6].

Theorem 1.6 is proved by first constructing solutions to an approximate system for every $\varepsilon>0$ and then passing to the limit $\varepsilon \rightarrow 0$ for a suitable subsequence. The approximate system is derived by replacing $\langle\delta V(t), \cdot\rangle$ by $\left\langle\delta V(t), \Psi_{\varepsilon} \cdot\right\rangle$ in (1.14) and replacing $v \cdot \nabla \chi$ by $\Psi_{\varepsilon} v \cdot \nabla \chi$ in (1.10), where $\Psi_{\varepsilon}$ is a suitable smoothing operator. This preserves the energy estimate. Moreover, the convective term in (1.14) is smoothed suitably. Using the same approximation scheme we extend the result of Nouri and Poupaud [17] on existence of weak solution of a two-phase flow of Newtonian fluids $\left(q=2\right.$ and $\left.v(j, s)=v_{j}\right)$ to a class of non-Newtonian fluids:

THEOREM 1.9 (Existence of weak solution, $\kappa=0$ ) Let $d=2,3$, let $q \geqslant 2 d /(d+2)+1$ or $q=2$ and $v(j, s)=v_{j}$, and let Assumption 1.1 hold. Moreover, let $v_{0} \in L_{\sigma}^{2}(\Omega), \chi_{0} \in L^{\infty}(\Omega ;\{0,1\})$, $f \in L^{q^{\prime}}\left(0, \infty ; V_{q}(\Omega)^{\prime}\right)$. Then there are $v \in L^{\infty}\left(0, \infty ; L_{\sigma}^{2}(\Omega)\right) \cap L^{q}\left(0, \infty ; V_{q}(\Omega)\right)$ and $\chi \in$ $L^{\infty}(Q ;\{0,1\}), Q:=\Omega \times(0, \infty)$, that are a weak solution of the two-phase flow without surface tension in the sense that

$$
-\left(v, \partial_{t} \varphi\right)_{Q}-\left(v_{0}, \varphi(0)\right)_{\Omega}-(v \otimes v, \nabla \varphi)_{Q}+(S(\chi, D v), D \varphi)_{Q}=\langle f, \varphi\rangle
$$

for all $\varphi \in C_{(0)}^{\infty}(\Omega \times[0, \infty))^{d}$ with $\operatorname{div} \varphi=0$, where $\chi$ is the unique renormalized solution of the transport equation of (1.10)-(1.11), and (1.17) holds for almost all $t>0$ with $\kappa=0$.

REMARK 1.10 In the case of a two-phase flow for the Stokes equation, i.e. the convective term $v \cdot \nabla v$ is neglected and (1.21) is replaced by

$$
-\left(v, \partial_{t} \varphi\right)_{Q}-\left(v_{0}, \varphi(0)\right)_{\Omega}+(S(\chi, D u), D \varphi)_{Q}=\langle f, \varphi\rangle,
$$

the same result as above holds for all $q>2 d /(d+2)$. Comments on the prove are given in Remark 5.5 below.

The structure of the article is as follows: After studying the necessary preliminaries in Section 2 , we first prove Proposition 1.5 in Section 3. Then we introduce the approximate system for the two-phase flow in Section 4 and prove existence of solutions for it. Using these solutions we pass to the limit in Section 5 and prove Theorems 1.6 and 1.9 . Finally in the appendix, we present a rectifiability criterion for the varifold in the two-phase flow, which is based on a new rectifiability result for varifolds due to Luckhaus [14].

\section{Preliminaries}

\subsection{Notation}

The set of all symmetric $d \times d$-matrices is denoted by $\mathbb{R}_{\text {sym }}^{d \times d}$. For $A, B \in \mathbb{R}_{\text {sym }}^{d \times d}$ we write $A: B=$ $\operatorname{Tr}(A B)$ and $|A|=\sqrt{A: A}$, where $\operatorname{Tr}$ denotes the trace of matrices. Given $a \in \mathbb{R}^{d}$ we define $a \otimes a \in \mathbb{R}_{\mathrm{sym}}^{d \times d}$ as the matrix with entries $a_{i} a_{j}, i, j=1, \ldots, d$. 
The dual of a topological vector space $V$ is denoted by $V^{\prime}$. If $v \in V$ and $v^{\prime} \in V^{\prime}$, then $\left\langle v, v^{\prime}\right\rangle \equiv$ $\left\langle v, v^{\prime}\right\rangle_{V, V^{\prime}}:=v^{\prime}(v)$ denotes the duality product. If $A: V \rightarrow W$ is a continuous linear operator, $A^{\prime}: W^{\prime} \rightarrow V^{\prime}$ denotes its adjoint.

For a given set $A \subset \mathbb{R}^{d}$, we define its $\varepsilon$-neighborhood $A_{\varepsilon}, \varepsilon>0$, as $A_{\varepsilon}=\bigcup_{x \in A} B_{\varepsilon}(x)$. Moreover, for given compact sets $A, B \subset \mathbb{R}^{d}$ the Hausdorff distance is defined as

$$
d_{H}(A, B)=\inf \left\{\varepsilon>0: A \subseteq B_{\varepsilon} \text { and } B \subseteq A_{\varepsilon}\right\} .
$$

If $A \subset \mathbb{R}^{d}$ is a compact set, then $\mathcal{K}(A)=\{B \subseteq A: B$ closed $\}$ equipped with the Hausdorff distance is a compact metric space (cf. e.g. [7, Proposition 2.4.4]).

\subsection{Function spaces}

Spaces of integrable functions. If $M \subseteq \mathbb{R}^{d}$ is measurable, then $L^{q}(M), 1 \leqslant q \leqslant \infty$, denotes the usual Lebesgue space and $\|\cdot\|_{q}$ its norm. Moreover, $L^{q}(M ; X)$ denotes its vector-valued variant of strongly measurable $q$-integrable (or essentially bounded) functions, where $X$ is a Banach space. More generally, if $X$ is a Fréchet space, then $f \in L^{q}(M ; X)$ if $f$ is strongly measurable and $q$-integrable/essentially bounded with respect to all the seminorms of $X$. For a subset $N \subset X$ we denote by $L^{q}(M ; N)$ the set of all $f \in L^{q}(M ; X)$ with $f(x) \in N$ for almost all $x \in M$. Furthermore, $f \in L_{\mathrm{loc}}^{q}([0, \infty) ; X)$ if and only if $f \in L^{q}(0, T ; X)$ for every $T>0$. If $\Omega \subseteq \mathbb{R}^{d}$ is a domain, then $f \in L_{\text {loc }}^{q}(\bar{\Omega})$ if and only if $f \in L^{q}(\Omega \cap B)$ for every ball $B$ with $B \cap \Omega \neq \emptyset$. For any measurable set $A \subset \mathbb{R}^{d}, \chi_{A}$ denotes its characteristic function.

Recall that, if $X$ is a Banach space with the Radon-Nikodym property, then

$$
L^{q}(M ; X)^{\prime}=L^{q^{\prime}}\left(M ; X^{\prime}\right) \quad \text { for every } 1 \leqslant q<\infty
$$

by means of the duality product

$$
\langle f, g\rangle=\int_{M}\langle f(x), g(x)\rangle \mathrm{d} x
$$

for $f \in L^{q}(M ; X), g \in L^{q^{\prime}}\left(M ; X^{\prime}\right)$. If $X$ is reflexive or $X^{\prime}$ is separable, then $X$ has the RadonNikodym property (cf. Diestel and Uhl [8]).

Moreover, recall the lemma of Aubin-Lions: If $X_{0} \hookrightarrow \hookrightarrow X_{1} \hookrightarrow X_{2}$ are Banach spaces, $1<$ $p<\infty, 1 \leqslant q<\infty$, and $I \subset \mathbb{R}$ is a bounded interval, then

$$
\left\{v \in L^{p}\left(I ; X_{0}\right): \frac{\mathrm{d} v}{\mathrm{~d} t} \in L^{q}\left(I ; X_{2}\right)\right\} \hookrightarrow \hookrightarrow L^{p}\left(I ; X_{1}\right) .
$$

See J.-L. Lions [13] for the case $q>1$ and Simon [26] or Roubíček [22] for $q=1$.

Furthermore, we note that, if $Y=X^{\prime}$ is a dual space, then $L_{\omega}^{\infty}(Q ; Y)$ for open $Q \subseteq \mathbb{R}^{N}$ is defined as all weak-* measurable functions $v: Q \rightarrow Y$, i.e.,

$$
x \mapsto\left\langle v_{x}, F(x, \cdot)\right\rangle=\left\langle v_{x}, F(x, \cdot)\right\rangle_{X^{\prime}, X}
$$

is measurable for each $F \in L^{1}(Q ; X)$, such that

$$
\|v\|_{L_{\omega}^{\infty}(Q ; Y)}:=\underset{x \in Q}{\operatorname{ess} \sup }\left\|v_{x}\right\|_{Y}<\infty .
$$


Sobolev and Bessel potential spaces. $W_{q}^{m}(\Omega), m \in \mathbb{N}_{0}, 1 \leqslant q \leqslant \infty$, denotes the usual $L^{q}$ Sobolev space, $W_{q, \text { loc }}^{m}(\bar{\Omega})$ its local version, $W_{q, 0}^{m}(\Omega)$ the closure of $C_{0}^{\infty}(\Omega)$ in $W_{q}^{m}(\Omega), W_{q}^{-m}(\Omega)=$ $\left(W_{q^{\prime}, 0}^{m}(\Omega)\right)^{\prime}$, and $f \in W_{q, \text { loc }}^{-m}(\bar{\Omega})$ if $f \in W_{q}^{-m}(\Omega \cap B)$ for every ball $B \subset \mathbb{R}^{d}$. The $L^{2}$-Bessel potential spaces are denoted by $H^{s}(\Omega), s \in \mathbb{R}$, defined as the restrictions of distributions in $H^{s}\left(\mathbb{R}^{d}\right)$ to $\Omega$ (cf. Triebel [34, Section 4.2.1]). Finally, $\dot{W}_{q}^{1}(\Omega)=\left\{f \in L_{\mathrm{loc}}^{q}(\bar{\Omega}): \nabla f \in L^{q}(\Omega)\right\}$, normed in the obvious way, denotes the homogeneous Sobolev space of first order, where functions differing by a constant are identified.

Spaces of continuous functions. The usual spaces of continuous, Hölder continuous, $k$-times differentiable and smooth functions on an open or closed set $A$ are denoted by $C(A), C^{\alpha}(A)$, $0<\alpha \leqslant 1, C^{k}(A)$, and $C^{\infty}(A)$, respectively, Furthermore, $C_{0}^{\infty}(\Omega) \equiv \mathcal{D}(\Omega)$ denotes the space of smooth and compactly supported functions on $\Omega$ and $C_{0}(\Omega), C_{0}^{k}(\Omega)$ denote the closures of $C_{0}^{\infty}(\Omega)$ in the corresponding norms. Moreover, if $A \subset \mathbb{R}^{d}$ is a set, then

$$
C_{(0)}^{\infty}(A)=\left\{f: A \rightarrow \mathbb{R}: f=\left.F\right|_{A}, F \in C_{0}^{\infty}\left(\mathbb{R}^{d}\right), \operatorname{supp} f \subseteq A\right\}
$$

equipped with the quotient topology. If $A$ is an open set, a subscript $b$ as in $C_{b}^{k}\left(\mathbb{R}^{d}\right)$ indicates that the functions and their derivatives are required to be bounded.

Spaces of solenoidal functions. In the following $C_{0, \sigma}^{\infty}(\Omega)$ denotes the space of all divergence free vector fields in $C_{0}^{\infty}(\Omega)^{d}$ and $L_{\sigma}^{q}(\Omega)$ is its closure in the $L^{q}$-norm. The corresponding Helmholtz projection is denoted by $P_{L_{\sigma}^{q}}$ or just $P_{\sigma}$ (cf. e.g. Simader and Sohr [25]).

Finally, recall that $V_{q}\left(\mathbb{R}^{d}\right)=\left\{v \in \dot{W}_{q}^{1}\left(\mathbb{R}^{d}\right)^{d}: \operatorname{div} f=0\right\}$ and $V_{q}(\Omega)=W_{q, 0}^{1}(\Omega) \cap L_{\sigma}^{q}(\Omega)$ if $\Omega$ is a bounded domain. In both cases $V_{q}$ will be normed by $\|v\|_{V_{q}(\Omega)}=\|D v\|_{L^{q}(\Omega)}$. By Korn's inequality this norm is equivalent to the standard norms.

Spaces of measures and functions of bounded variations. These spaces are defined at the beginning of Sections 2.3 and 2.4 .

\subsection{Measures, disintegration and Young measures}

Let $X$ be a locally compact separable metric space and let $C_{0}\left(X ; \mathbb{R}^{m}\right)$ be the closure of the compactly supported continuous functions $f: X \rightarrow \mathbb{R}^{m}, m \in \mathbb{N}$, in the supremum norm. Moreover, denote by $\mathcal{M}\left(X ; \mathbb{R}^{m}\right)$ the space of all finite $\mathbb{R}^{m}$-valued Radon measures, $\mathcal{M}(X):=\mathcal{M}(X ; \mathbb{R})$, and let $\operatorname{Prob}(X)$ denote the space of all probability measure on $X$. Then by the Riesz representation theorem $\mathcal{M}\left(X ; \mathbb{R}^{m}\right)=C_{0}\left(X ; \mathbb{R}^{m}\right)^{\prime}$ (cf. e.g. Ambrosio et al. [2, Theorem 1.54]). Given $\mu \in$ $\mathcal{M}\left(X ; \mathbb{R}^{m}\right)$ the total variation measure is defined by

$$
|\mu|(A)=\sup \left\{\sum_{k=0}^{\infty}\left|\mu\left(A_{k}\right)\right|: A_{k} \in \mathcal{B}(X) \text { pairwise disjoint, } A=\bigcup_{k=0}^{\infty} A_{k}\right\}
$$

for every $A \in \mathcal{B}(X)$, where $\mathcal{B}(X)$ denotes the $\sigma$-algebra of Borel subsets of $X$. Then by [2, Proposition 1.47],

$$
|\mu|(A)=\sup \left\{\int_{X} f(x) \mathrm{d} \mu(x): f \in C_{0}\left(X ; \mathbb{R}^{m}\right), \operatorname{supp} f \subset A,\|f\|_{\infty} \leqslant 1\right\}
$$


for every open set $A \subseteq X$. The restriction of a measure $\mu$ to a $\mu$-measurable set $A$ is denoted by $\left(\mu\lfloor A)(B)=\mu(A \cap B)\right.$. Finally, the $s$-dimensional Hausdorff measure on $\mathbb{R}^{d}, 0 \leqslant s \leqslant d$, is denoted by $\mathcal{H}^{s}$.

Now let $U \subset \mathbb{R}^{N}, V \subset \mathbb{R}^{M}$ be open sets and let $v \in \mathcal{M}\left(U \times V ; \mathbb{R}^{m}\right)$. Moreover, we set $\mu(A)=$ $|\nu|(A \times V)$. Then by the disintegration theorem (cf. [2, Theorem 2.28]), there is a $\mu$-measurable mapping $x \mapsto v_{x}$ such that $\left|v_{x}\right| \in \operatorname{Prob}(V)$ for $\mu$-a.e. $x \in U$ and for any $f \in L^{1}(U \times V,|v|)$,

$$
\begin{aligned}
& f(x, \cdot) \in L^{1}\left(V,\left|v_{x}\right|\right) \quad \text { for } \mu \text {-a.e. } x \in U, \\
& x \mapsto \int_{V} f(x, y) \mathrm{d} v_{x}(y) \in L^{1}(U, \mu), \\
& \int_{U \times V} f(x, y) \mathrm{d} v(x, y)=\int_{U}\left(\int_{V} f(x, y) \mathrm{d} v_{x}(y)\right) \mathrm{d} \mu(x) .
\end{aligned}
$$

Obviously, if $v \in \mathcal{M}(U \times V)$ is a non-negative measure, then $v_{x}=\left|v_{x}\right| \in \operatorname{Prob}(V)$ for $\mu$-a.e. $x \in U$.

We need the following version of the fundamental theorem on Young measures:

THEOREM 2.1 Let $Q \subset \mathbb{R}^{N}$ be an open set and let $z_{j} \in L^{p}\left(Q ; \mathbb{R}^{m}\right), 1<p<\infty$, be a bounded sequence. Then there is a subsequence still denoted by $z_{j}$ and a weak-* measurable function $x \mapsto$ $v_{x} \in \operatorname{Prob}\left(\mathbb{R}^{m}\right)$ such that for every continuous $\tau: \mathbb{R}^{m} \rightarrow \mathbb{R}$ satisfying the growth condition

$$
|\tau(\xi)| \leqslant C(1+|\xi|)^{p-1} \quad \text { for all } \xi \in \mathbb{R}^{d}
$$

for some $C>0$ we have

$$
\tau\left(z^{j}\right) \rightarrow \bar{\tau} \quad \text { in } L^{p^{\prime}}(Q) \text { as } j \rightarrow \infty
$$

where $\bar{\tau}=\left\langle v_{x}, \tau\right\rangle$ for almost all $x \in Q$.

Proof. The result immediately follows from Corollary 2.10 in Málek et al. [16, Section 4.2] by choosing $q=p-1, r=p / q=p^{\prime}$. Moreover, we note that the restriction to a bounded set in that corollary is only needed if $1<r<p / q$ as can be easily seen in the proof.

Finally, recall the generalized Jensen inequality: Let $g: \mathbb{R}^{N} \rightarrow \mathbb{R}$ be a strictly convex function and let $\mu$ be a probability measure on $\mathbb{R}^{N}$ such that id and $|g|$ are $\mu$-integrable. Then

$$
g\left(\int x \mathrm{~d} \mu(x)\right) \leqslant \int_{\mathbb{R}^{N}} g(x) \mathrm{d} \mu(x),
$$

with equality if and only if $\mu$ is a Dirac measure (cf. e.g. [16, Lemma 2.27, Chapter III] and its proof).

\section{$2.4 \quad B V$-functions and varifolds}

Let $U \subseteq \mathbb{R}^{d}$ be an open set. Recall that

$$
\begin{aligned}
B V(U) & =\left\{f \in L^{1}(U): \nabla f \in \mathcal{M}\left(U ; \mathbb{R}^{d}\right)\right\}, \\
\|f\|_{B V(U)} & =\|f\|_{L^{1}(U)}+\|\nabla f\|_{\mathcal{M}\left(U ; \mathbb{R}^{d}\right)},
\end{aligned}
$$

where $\nabla f$ denotes the distributional derivative. Moreover, $B V(U ;\{0,1\})$ denotes the set of all $\chi \in B V(U)$ such that $\chi(x) \in\{0,1\}$ for almost all $x \in U$. 
Moreover, a set $E \subseteq U$ is said to have finite perimeter in $U$ if $\chi_{E} \in B V(U)$. Then by the structure theorem on sets of finite perimeter $\left|\nabla \chi_{E}\right|=\mathcal{H}^{d-1}\left\lfloor\partial^{*} E\right.$, where $\partial^{*} E$ is the so-called reduced boundary of $E$ and

$$
-\left\langle\nabla \chi_{E}, \varphi\right\rangle=\int_{E} \operatorname{div} \varphi \mathrm{d} x=\int_{\partial^{*} E} \varphi \cdot n_{E} \mathrm{~d} \mathcal{H}^{d-1},
$$

where $n_{E}(x)=-\nabla \chi_{E} /\left|\nabla \chi_{E}\right|$ (cf. e.g. [2]). Note that, if $E$ is a domain with $C^{1}$-boundary, then $\partial^{*} E=\partial \Omega$ and $n_{E}$ coincides with the exterior unit normal.

For a set $E$ of finite perimeter in $U$ we define the mean curvature functional associated to $\partial^{*} E$ as

$$
\left\langle H_{\partial^{*} E}, \varphi\right\rangle \equiv\left\langle H_{\chi_{E}}, \varphi\right\rangle:=-\int_{\partial^{*} E} \operatorname{Tr}\left(P_{\tau} \nabla \varphi\right) \mathrm{d} \mathcal{H}^{d-1}, \quad \varphi \in C_{0}^{1}(\Omega)^{d},
$$

where $P_{\tau}=I-n_{E}(x) \otimes n_{E}(x)$.

A general $(d-1)$-varifold $\widetilde{V}$ is simply a measure $\widetilde{V} \in \mathcal{M}\left(U \times G_{d-1}\right)$, where $G_{d-1}$ is the space of all unoriented $(d-1)$-dimensional linear subspaces of $\mathbb{R}^{d}$ (cf. Simon [27]). The first variation $\delta \widetilde{V}$ of a general varifold $\widetilde{V}$ is defined as

$$
\langle\delta \tilde{V}, \psi\rangle=\int_{U \times G_{d-1}} P_{T}: \nabla \psi \mathrm{d} \widetilde{V}(x, T) \quad \text { for } \psi \in C_{0}^{1}(U)^{d},
$$

where $P_{T}$ denotes the orthogonal projection onto $T \in G_{d-1}$. Note that general varifolds are unoriented and that $G_{d-1} \cong \mathbb{S}^{d-1} /\{x \equiv-x\}$. If $E$ is a set of finite perimeter in $U$, then its reduced boundary can be identified with the varifold defined by

$$
\left\langle\widetilde{V}_{\partial^{*} E}, \varphi\right\rangle=\int_{\partial^{*} E} \varphi\left(x,\left[n_{E}(x)\right]\right) \mathrm{d} \mathcal{H}^{d-1} \quad \text { for all } \varphi \in C_{0}\left(U \times G_{d-1}\right),
$$

where $\left[n_{E}(x)\right]$ denotes the $(d-1)$-dimensional subspace of $\mathbb{R}^{d}$ with normal $n_{E}(x)$. Then

$$
\left\langle\delta \widetilde{V}_{\partial^{*} E}, \psi\right\rangle=-\left\langle H_{\partial^{*} E}, \psi\right\rangle \quad \text { for all } \psi \in C_{0}^{1}(U)^{d} .
$$

Hence the mean curvature functional associated to $\partial^{*} E$ can be recovered from the general varifold associated to $\partial^{*} E$. But this is not the case for $\nabla \chi_{E}=-n_{E} \mathcal{H}^{d-1}\left\lfloor\partial^{*} E\right.$ since general varifolds do not take orientation into account. Therefore we define an oriented general $(d-1)$-varifold as a non-negative measure $V \in \mathcal{M}\left(U \times \mathbb{S}^{d-1}\right)$ as was done for example by Soner [31, Section 2.3]. By disintegration $V$ can be written in the form

$$
\langle V, \varphi\rangle=\int_{U} \int_{\mathbb{S}^{d-1}} \varphi(x, s) \mathrm{d} V_{x}(s) \mathrm{d}|V|(x), \quad \varphi \in C_{0}\left(U \times \mathbb{S}^{d-1}\right) .
$$

Obviously, every oriented general varifold $V$ induces an (unoriented) general varifold $\widetilde{V}$ by

$$
\langle\widetilde{V}, \varphi\rangle=\int_{U \times \mathbb{S}^{d-1}} \varphi(x,[s]) \mathrm{d} V(x, s), \quad \varphi \in C_{0}\left(U \times G_{d-1}\right),
$$

where again $[s]$ denotes the $(d-1)$-dimensional linear subspace of $\mathbb{R}^{d}$ with $s$ as normal. Now, if $E$ has finite perimeter in $U$, then we associate to $\partial^{*} E$ the oriented general varifold $V_{\partial^{*} E}$ defined by

$$
\left\langle V_{\partial^{*} E}, \varphi\right\rangle=\int_{\partial^{*} E} \varphi\left(x, n_{E}(x)\right) \mathrm{d} \mathcal{H}^{d-1} \quad \text { for all } \varphi \in C_{0}\left(U \times \mathbb{S}^{d-1}\right) .
$$

Note that this corresponds to the choice $|V|=\mathcal{H}^{d-1}\left\lfloor\partial^{*} E\right.$ and $V_{x}=\delta_{n_{E}(x)}$ in 2.6. 
Now we recover $\nabla \chi_{E}$ from $V=V_{\partial^{*} E}$ by choosing $\varphi(x, s)=s \cdot \psi(x)$ with $\psi \in C_{0}\left(U ; \mathbb{R}^{d}\right)$ :

$$
\left\langle V_{\partial^{*} E}, \varphi\right\rangle=\int_{U \times \mathbb{S}^{d-1}} s \cdot \psi(x) \mathrm{d} V(x, s)=\int_{\partial^{*} E} \psi \cdot n_{E} \mathrm{~d} \mathcal{H}^{d-1}=-\left\langle\nabla \chi_{E}, \psi\right\rangle .
$$

Finally, let $\Gamma_{0} \subseteq \mathbb{R}^{d}$ be the boundary of a bounded $C^{1}$-domain $\Omega_{0}^{+}$with exterior normal vector field $n$ and let $X_{t}: \mathbb{R}^{d} \rightarrow \mathbb{R}^{d}, t>0$, be a family of $C^{1}$-diffeomorphisms depending in a continuously differentiable way on $t>0$ such that $\frac{\mathrm{d}}{\mathrm{d} t} X_{t}(x)=v\left(X_{t}(x), t\right)$ for a sufficiently smooth vector field $v$. Moreover, set $\Gamma_{t}=X_{t}\left(\Gamma_{0}\right)$ and $\Omega_{t}=X_{t}\left(\Omega_{0}^{+}\right), t>0$. Then one calculates that

$$
\frac{\mathrm{d}}{\mathrm{d} t} \int_{\Gamma_{t}} \varphi(x) \mathrm{d} \mathcal{H}^{d-1}(x)=\left\langle\delta V_{\Gamma_{t}}, \varphi v(t)\right\rangle+\int_{\Gamma_{t}} n \cdot \nabla \varphi(x) n \cdot v(x, t) \mathrm{d} \mathcal{H}^{d-1}(x)
$$

for every $\varphi \in C_{0}^{1}\left(\mathbb{R}^{d}\right)$, where $V_{\Gamma_{t}}$ denotes the general varifold associated to $\Gamma_{t}$ defined as above and $n$ is the exterior normal at $\Gamma_{t}=\partial \Omega_{t}$.

\subsection{Transport equation}

We consider weak solutions of the transport equation

$$
\begin{array}{rlrl}
\partial_{t} \chi+v \cdot \nabla \chi & =0 & & \text { in } Q_{T}, \\
\left.\chi\right|_{t=0}=\chi_{0} & & \text { in } \Omega,
\end{array}
$$

where $Q_{T}=\Omega \times(0, T), 0<T \leqslant \infty, \Omega=\mathbb{R}^{d}$ or $\Omega$ is a bounded Lipschitz domain, $v \in$ $L_{\text {loc }}^{2}\left([0, \infty) ; L_{\sigma}^{2}(\Omega)\right)$, and $\chi_{0} \in L^{\infty}(\Omega)$. Here a weak solution is a function $\chi \in L^{\infty}(Q)$ satisfying

$$
\int_{Q} \chi\left(\partial_{t} \varphi+v \cdot \nabla \varphi\right) \mathrm{d}(x, t)+\int_{\Omega} \chi_{0} \varphi(x, 0) \mathrm{d} x=0
$$

for all $\varphi \in C_{(0)}^{\infty}(\bar{\Omega} \times[0, T))$. Then we have

Proposition 2.2 For every $\chi_{0} \in L^{\infty}(\Omega)$ and $v \in L_{\text {loc }}^{2}\left([0, \infty) ; L_{\sigma}^{2}(\Omega)\right)$ there is a unique weak solution of 2.9]-2.10) with $T=\infty$. Moreover, this solution is a renormalized solution, i.e., $\beta(\chi)$ is a weak solution associated to the data $\beta\left(\chi_{0}\right)$ for any $\beta \in C^{1}(\mathbb{R})$. Furthermore, if $\chi_{0} \in M$ a.e. for some finite set $M$, then $\chi \in M$ a.e.

The proposition follows from Nouri, Poupaud and Demay [18, Theorem 4.1]. It essentially coincides with [17, Proposition 3.3]. These results are based on DiPerna and Lion's results on weak and renormalized solutions of the transport equation (cf. [9]). In that work $\operatorname{div} v=0$ or $\operatorname{div} v \in L^{\infty}$ is essentially used.

In order to construct approximate solutions of the two-phase flow with surface tension we use:

Lemma 2.3 Let $\chi_{0} \in B V\left(\mathbb{R}^{d} ;\{0,1\}\right)$ and let $v \in C\left([0, T] ; C_{b}^{2}\left(\mathbb{R}^{d}\right)^{d}\right)$, div $v=0, T>0$. Then there is a weak solution $\chi \in L^{\infty}\left(0, T ; B V\left(\mathbb{R}^{d} ;\{0,1\}\right)\right)$ of 2.9 2.10 . Moreover,

$$
\begin{aligned}
\|\chi\|_{L^{\infty}\left(0, T ; B V\left(\mathbb{R}^{d}\right)\right)} & \leqslant M\left(\|v\|_{C\left([0, T] ; C_{b}^{2}\left(\mathbb{R}^{d}\right)\right)}\right)\left\|\chi_{0}\right\|_{B V\left(\mathbb{R}^{d}\right)}, \\
\frac{\mathrm{d}}{\mathrm{d} t}|\nabla \chi(t)|\left(\mathbb{R}^{d}\right) & =-\left\langle H_{\chi(t)}, v(t)\right\rangle \quad \text { for all } t \in(0, T)
\end{aligned}
$$

for some continuous function $M$. 
Proof. The solution $\chi$ is constructed by the usual method of characteristics. Since $v \in$ $C\left([0, T] ; C_{b}^{1}\left(\mathbb{R}^{d}\right)^{d}\right)$ for every $x_{0} \in \mathbb{R}^{d}$ there is a unique solution $x\left(t ; x_{0}\right) \in C^{1}\left(0, \infty ; \mathbb{R}^{d}\right)$ of

$$
\begin{aligned}
\frac{\mathrm{d}}{\mathrm{d} t} x\left(t ; x_{0}\right) & =v\left(x\left(t ; x_{0}\right), t\right), \quad t>0, \\
x\left(0 ; x_{0}\right) & =x_{0},
\end{aligned}
$$

which is a trajectory along the vector field $v$. Note that, since $v$ is globally Lipschitz, the solution $x\left(t ; x_{0}\right)$ exists for all $t \in(0, T)$. Let $X\left(x_{0}, t\right):=x\left(t ; x_{0}\right)$ and let $X_{t}=X(\cdot, t)$ be the flow mapping. Then $X \in C^{1}\left([0, T] \times \mathbb{R}^{d}\right)$ by the usual $C^{1}$-dependence on the initial values and $X_{t}: \mathbb{R}^{d} \rightarrow \mathbb{R}^{d}$ is a $C^{1}$-diffeomorphism. Now define $\chi(x, t):=\chi_{0}\left(X_{t}^{-1} x\right)$. Then $\|\chi(\cdot, t)\|_{L^{1}\left(\mathbb{R}^{d}\right)}=\left\|\chi_{0}\right\|_{L^{1}\left(\mathbb{R}^{d}\right)}$ since det $D X_{t}(y)=\operatorname{det} D X_{0}(y)=1$ because of $\partial_{t} \operatorname{det} D X_{t}(y)=\operatorname{div} v\left(X_{t}(y, t)\right)=0$. In order to estimate $\chi \in L^{\infty}\left(0, T ; B V\left(\mathbb{R}^{d} ;\{0,1\}\right)\right)$, we use the fact that

$$
\begin{aligned}
\int_{\Omega} \chi(x, t) \operatorname{div} \psi(x) \mathrm{d} x & =\int_{\Omega} \chi_{0}(y) \operatorname{Tr}\left((\nabla \psi)\left(X_{t}(y)\right)\right) \mathrm{d} y \\
& =\int_{\Omega} \chi_{0}(y) \operatorname{Tr}(\nabla \widetilde{\psi}(y)) \mathrm{d} y-\int_{\Omega} \chi_{0}(y) \operatorname{Tr}\left(\nabla D X_{t}^{-T}\right) \psi\left(X_{t}(y)\right) \mathrm{d} y,
\end{aligned}
$$

where $\widetilde{\psi}(y)=D X_{t}^{-T} \psi\left(X_{t}(y)\right), \psi \in C_{0}^{1}\left(\mathbb{R}^{d}\right)^{d}$. Hence

$$
\sup _{t \in[0, T]}\left|\int_{\Omega} \chi(x, t) \operatorname{div} \psi(x) \mathrm{d} x\right| \leqslant M\left(\|v\|_{C\left([0, T] ; C_{b}^{2}\left(\mathbb{R}^{d}\right)\right)}\right)\left\|\chi_{0}\right\|_{B V\left(\mathbb{R}^{d}\right)}\|\psi\|_{C_{b}^{0}\left(\mathbb{R}^{d}\right)}
$$

for all $\psi \in C_{0}^{1}\left(\mathbb{R}^{d}\right)^{d}$ and $t>0$ and some continuous function $M$. Moreover, by standard calculations

$$
\begin{aligned}
\left(\chi, \partial_{t} \varphi\right)_{Q} & =\int_{0}^{\infty} \int_{\Omega} \chi_{0}(y) \partial_{t} \varphi\left(X_{t}(y), t\right) \mathrm{d} y \mathrm{~d} t \\
& =-\left(\chi_{0},\left.\varphi\right|_{t=0}\right)-\int_{0}^{\infty} \int_{\Omega} \chi_{0}(y) \nabla \varphi\left(X_{t}(y), t\right) \cdot v\left(X_{t}(y), t\right) \mathrm{d} y \mathrm{~d} t \\
& =-\left(\chi_{0},\left.\varphi\right|_{t=0}\right)-(\chi, v \cdot \nabla \varphi)_{Q}
\end{aligned}
$$

for all $\varphi \in C_{(0)}^{\infty}\left([0, \infty) \times \mathbb{R}^{d}\right)$. Hence $\chi$ is a weak solution of $2.97-\sqrt{2.10}$.

Finally, the last identity follows from 2.8 .

For the following we note that $C^{1}\left(\mathbb{R}^{d}\right)$ is equipped with the topology of locally uniform convergence of functions and their first order derivatives.

Lemma 2.4 Let $\chi_{0}=\chi_{\Omega_{0}^{+}}$, where $\Omega_{0}^{+}$is a bounded $C^{1}$-domain. Moreover, let $u_{k}, u \in$ $C\left([0, T] ; C_{b}^{2}\left(\mathbb{R}^{d}\right)^{d}\right)$ be such that $u_{k} \rightarrow u$ in $C\left([0, T] ; C^{1}\left(\mathbb{R}^{d}\right)^{d}\right)$ as $k \rightarrow \infty$. Then for any $f \in C_{b}^{0}\left(\mathbb{R}^{d} \times \mathbb{S}^{d-1}\right)$,

$$
\lim _{k \rightarrow \infty} \int_{\Gamma_{u_{k}}(t)} f\left(x, n_{x}\right) \mathrm{d} \mathcal{H}^{d-1}(x)=\int_{\Gamma_{u}(t)} f\left(x, n_{x}\right) \mathrm{d} \mathcal{H}^{d-1}(x)
$$

uniformly in $t \in(0, T)$, where $\Gamma_{w}(t)=X_{w}(t)\left(\partial \Omega_{0}^{+}\right)$and $X_{w}(t)$ is the flow map obtained from 2.14 - 2.15) with $v=w$ as above. Finally, $\left\{\Gamma_{u_{k}}(t), \Gamma_{u}(t): k \in \mathbb{N}, t \in[0, T]\right\}$ is contained in a compact set. 
Proof. First of all, $X_{u_{k}} \in C^{1}\left([0, T] \times \mathbb{R}^{d}\right)$ and $X_{u_{k}} \rightarrow X_{u} \in C^{1}\left([0, T] \times B_{R}(0)\right), R>0$, by the usual $C^{1}$-dependence of solutions of ordinary differential equations on the data. Moreover, by construction $X_{u_{k}}(t): \mathbb{R}^{d} \rightarrow \mathbb{R}^{d}$ is bijective for any $t \in[0, T]$. Hence $X_{u_{k}}^{-1}(t): \mathbb{R}^{d} \rightarrow \mathbb{R}^{d}$ is continuously differentiable and $X_{u_{k}}^{-1}(t) \rightarrow X_{u}^{-1}(t)$ in $C^{1}\left(\mathbb{R}^{d}\right)$ for any $t \in[0, T]$. Using all this, the lemma can be proved by either introducing a local parameterization of $\partial \Omega_{0}^{+}$and using $X_{u_{k}}(t)$ and $X_{u}(t)$ to get suitable parameterizations of $\Gamma_{u_{k}}(t)$ and $\Gamma_{u}(t)$, or one can use the continuity theorem by Reshetnyak: Since $X_{u_{k}}(t) \rightarrow X_{u}(t)$ and $X_{u_{k}}^{-1}(t) \rightarrow X_{u}^{-1}(t)$ in $C^{1}\left(\mathbb{R}^{d}\right)$, it is an easy exercise to show $\mathcal{H}^{d-1}\left(\Gamma_{u_{k}}(t)\right) \rightarrow \mathcal{H}^{d-1}\left(\Gamma_{u}(t)\right)$. Moreover, if $\Omega_{k}^{+}(t)=X_{u_{k}}(t)\left(\Omega_{0}^{+}\right)$and $\Omega^{+}(t)=X_{u}(t)\left(\Omega_{0}^{+}\right)$, then

$$
\left\langle\nabla \chi_{\Omega_{k}^{+}(t)}, \varphi\right\rangle=-\int_{\Omega_{0}^{+}} \operatorname{div} \varphi\left(X_{u_{k}}(t)\right) \mathrm{d} x \rightarrow-\int_{\Omega_{0}^{+}} \operatorname{div} \varphi\left(X_{u}(t)\right) \mathrm{d} x=\left\langle\nabla \chi_{\Omega^{+}(t)}, \varphi\right\rangle
$$

as $k \rightarrow \infty$ for all $\varphi \in C_{0}^{1}\left(\mathbb{R}^{d}\right)^{d}$. This implies $\nabla \chi_{\Omega_{k}^{+}(t)} \rightarrow^{*} \nabla \chi_{\Omega^{+}(t)}$ in $\mathcal{M}\left(\mathbb{R}^{d}\right)$ since $\left\|\nabla \chi_{u_{k}}\right\|_{\mathcal{M}}=$ $\mathcal{H}^{d-1}\left(\Gamma_{u_{k}}(t)\right)$ are uniformly bounded and $C_{0}^{1}\left(\mathbb{R}^{d}\right)$ is dense in $C_{0}\left(\mathbb{R}^{d}\right)$. Therefore one can apply [2. Theorem 2.39] to the vector measures $\nabla \chi_{\Omega_{k}^{+}(t)}$ and $\nabla \chi_{\Omega^{+}(t)}$ to show 2.16 .

Finally, the last statement is an easy consequence of the fact that $X_{u_{k}} \rightarrow X_{u}$ in $C^{1}\left([0, T] \times \mathbb{R}^{d}\right)$ and the compactness of $\partial \Omega_{0}^{+}$.

LEMMA 2.5 Let $u_{k}, u \in L^{1}\left(0, T ; L_{\sigma}^{2}(\Omega)\right), k \in \mathbb{N}$, for some $T>0$ such that $u_{k} \rightarrow u$ in $L^{1}\left(0, T ; L_{\text {loc }}^{2}(\bar{\Omega})\right)$. Moreover, let $\chi_{k}, \chi \in L^{\infty}\left(Q_{T}\right)$ be the solutions of $2.9-\sqrt{2.10}$ with $v=u_{k}, u$, resp., and $\chi_{0}=\chi_{E}$ for some fixed measurable set $E$. Then $\chi_{k} \rightarrow^{*} \chi$ in $L^{\infty}\left(Q_{T}\right)$ and $\chi_{k} \rightarrow \chi$ in $L^{p}\left(Q_{T}\right)$ for every $p<\infty$.

Proof. First of all, since $\chi_{k} \in L^{\infty}\left(Q_{T}\right)$ are uniformly bounded, $\chi_{k_{j}} \rightarrow^{*} \tilde{\chi}_{0}$ in $L^{\infty}\left(Q_{T}\right)$ as $j \rightarrow \infty$ for some $\tilde{\chi}_{0} \in L^{\infty}\left(Q_{T}\right)$ and some suitable subsequence. Since $u_{k} \rightarrow u$ in $L^{1}\left(0, T ; L_{\text {loc }}^{2}(\Omega)\right)$, $u_{k} \cdot \nabla \varphi \rightarrow u \cdot \nabla \varphi$ in $L^{1}\left(0, T ; L^{2}(\Omega)\right)$ for any $\varphi \in C_{(0)}^{\infty}(\bar{\Omega} \times[0, \infty))$. Thus $\tilde{\chi}_{0}$ solves the transport equation with $v=u$. Hence

$$
\left\|\chi_{k_{j}}\right\|_{L^{q}\left(Q_{T}\right)}^{q}=T|E|=\left\|\tilde{\chi}_{0}\right\|_{q}^{q}
$$

for every $1 \leqslant q<\infty$. Thus $\chi_{k_{j}} \rightarrow \tilde{\chi}_{0}$ in $L^{q}\left(Q_{T}\right)$ strongly. In particular, this implies $\tilde{\chi}_{0} \in\{0,1\}$ almost everywhere. Therefore $\widetilde{\chi}_{0}$ coincides with the unique renormalized solution $\chi_{0}$. Since this argument holds for any subsequence, the sequence $\left(\chi_{k}\right)_{k \in \mathbb{N}}$ converges itself.

\subsection{A convergence result for monotone non-linearities}

In order to construct weak solutions in the case $\kappa=0$, we will use the following result:

ThEOREM 2.6 (Świerczewska [32, Lemma A.1]) Let $E \subset \mathbb{R}^{d}$ be a measurable set of finite measure and let $A: E \times \mathbb{R}^{m} \times \mathbb{R}^{N} \rightarrow \mathbb{R}^{N}$ be a function such that

1. $A(x, s, \xi)$ is a Carathéodory function with respect to $x$ and $(s, \xi)$, i.e., $A$ is measurable with respect to $x$ and continuous with respect to $(s, \xi)$.

2. $A(x, s, \xi)$ is strictly monotone with respect to $\xi$ : For almost all $x \in E$ and all $s \in \mathbb{R}^{m}$ and $\xi_{1}, \xi_{2} \in \mathbb{R}^{N}, \xi_{1} \neq \xi_{2}$,

$$
\left(A\left(x, s, \xi_{1}\right)-A\left(x, s, \xi_{2}\right)\right) \cdot\left(\xi_{1}-\xi_{2}\right)>0 .
$$


3. There are $q>1$ and $c_{1}, c_{2}>0$ such that

$$
A(x, s, \xi) \cdot \xi \geqslant c_{1}|\xi|^{q}, \quad|A(x, s, \xi)| \leqslant c_{2}|\xi|^{q-1}
$$

for almost all $x \in E$ and all $(s, \xi) \in \mathbb{R}^{m} \times \mathbb{R}^{N}$.

Moreover, let $y_{n}: E \rightarrow \mathbb{R}^{m}$ and $z_{n}: E \rightarrow \mathbb{R}^{N}$ be a sequence of measurable functions such that $y_{n} \rightarrow y$ a.e. in $E, z_{n} \rightarrow z$ in $L^{q}(E)$ and $A\left(x, y_{n}, z_{n}\right) \rightarrow \bar{A}$ in $L^{q^{\prime}}(E)$ as $n \rightarrow \infty$. Then

$$
\limsup _{n \rightarrow \infty} \int_{E} A\left(x, y_{n}, z_{n}\right) \cdot z_{n} \mathrm{~d} x \leqslant \int_{E} \bar{A} \cdot z \mathrm{~d} x
$$

implies $z_{n} \rightarrow z$ in measure as $n \rightarrow \infty$.

In the following we will apply the theorem to the case $x \in \Omega, s \in \mathbb{R}, \xi=\lambda \in \mathbb{R}_{\mathrm{sym}}^{d \times d}$, and $A(x, s, \xi)=S(s, \lambda)$. In this case Assumption 1.1 implies the assumptions of the theorem.

\section{Proof of Proposition 1.5}

First of all, we note that by $(1.12), \lambda \mapsto S(l, \lambda): \lambda, \lambda \in \mathbb{R}_{\mathrm{sym}}^{d \times d}$, is a strictly convex function for every $l \in[0,1]$.

First assume that $\|V(t)\|=\|\nabla \chi(t)\|$ for almost all $t \in(0, T)$. We will prove that $V_{x}(t)=\delta_{n(x, t)}$ for $|V(t)|$-almost every $x \in \mathbb{R}^{d}$ and almost every $t \in(0, \infty)$, where $n(x, t)=-\frac{\nabla \chi(t)}{|\nabla \chi(t)|}(x)$. From (1.15) we know that

$$
\int_{\Omega} \int_{\mathbb{S}^{d-1}} s \cdot \psi(x) \mathrm{d} V_{x}(t) \mathrm{d}|V(t)|=\int_{\Omega} n(x, t) \cdot \psi(x) \mathrm{d}|\nabla \chi(t)|
$$

for all $\psi \in C_{0}(\Omega)^{d}$. Hence by 2.2$),|\nabla \chi(t)|(A) \leqslant|V(t)|(A)$ for every open $A \subset \Omega$. Thus $|\nabla \chi(t)|$ is absolutely continuous with respect to $|V(t)|$ and

$$
|\nabla \chi(t)|(A)=\int_{A} \theta_{t}(x) \mathrm{d}|V(t)|(x)
$$

with some $|V(t)|$-measurable function $\theta_{t}: \mathbb{R}^{d} \rightarrow[0, \infty)$ and $\theta_{t}(x) \leqslant 1$ for $|V(t)|$-almost all $x$ in $\mathbb{R}^{d}$. But, since $\|\nabla \chi(t)\|=|\nabla \chi(t)|\left(\mathbb{R}^{d}\right)=|V(t)|\left(\mathbb{R}^{d}\right)=\|V(t)\|$, we conclude that $\theta_{t}(x)=1$ almost everywhere and $|V(t)|=|\nabla \chi(t)|$ as measures. Therefore 1.15 yields

$$
\int_{\mathbb{S}^{d-1}} s \mathrm{~d} V_{x}(t)(s)=n(x, t) \quad \text { for }|V(t)| \text {-almost all } x \in \Omega .
$$

Thus

$$
\frac{1}{2} \int|s-n(x, t)|^{2} \mathrm{~d} V_{x}(t)(s)=1-n(x, t) \cdot \int_{\mathbb{S}^{d-1}} s \mathrm{~d} V_{x}(t)(s)=0
$$

for $|V(t)|$-almost all $x \in \Omega$, which implies that $V_{x}(t)=\delta_{n(x, t)}$ for $|V(t)|$-almost every $x \in \mathbb{R}^{d}$.

If $(v, \chi)$ satisfies 1.20 , then necessarily $\|\nabla \chi(t)\|_{\mathcal{M}}=\|V(t)\|_{\mathcal{M}}$ for almost all $t>0$ because of (1.17) and 1.18). Hence the first part implies that $V_{x}(t)=\delta_{n(x, t)}$, which yields $\delta V(t)=-H_{\chi(t)}$. Moreover, by (2.4) and (1.16),

$$
S(\chi(x, t), D v(x, t)): D v(x, t) \leqslant \int S(\chi(x, t), \lambda): \lambda \mathrm{d} \mu_{x, t}(\lambda)
$$


with equality if and only if $\mu_{x, t}$ is a Dirac measure. (Note that $\int S(\chi(x, t), \lambda): \lambda \mathrm{d} \mu_{x, t}(\lambda)<\infty$ for almost all $(x, t) \in Q$ by $(1.17)$.) On the other hand, by (1.17)- 1.20$)$,

$$
\int_{Q_{t}} S(\chi(x, t), D v(x, t)): D v(x, t) \mathrm{d}(x, t)=\int_{Q_{t}} \int_{\mathbb{R}_{\mathrm{sym}}^{d \times d}} S(\chi, \lambda): \lambda \mathrm{d} \mu_{x, t}(\lambda) \mathrm{d}(x, t)
$$

for almost all $t>0$. Hence $\mu_{x, t}$ is a Dirac measure for almost all $(x, t) \in Q$, which implies that $\mu_{x, t}=\delta_{D v(x, t)}$ because of $(1.16)$. Altogether, we have proved that $(v, \chi)$ is a weak solution. The same argument also shows that $(v, \chi, V)$ is a varifold solution if 1.20$)$ holds with $\|\nabla \chi(t)\|$ replaced by $\|V(t)\|$.

Finally, if $q>d$, then 2.11 and the fact that $\chi \in L^{\infty}\left(0, \infty ; B V\left(\mathbb{R}^{d}\right)\right)$ yield

$$
\begin{aligned}
-\left(\chi, \operatorname{div}_{x, t} \psi\right) & =\int_{0}^{\infty} \int \psi^{\prime}(x, t) \mathrm{d} \nabla \chi(t) \mathrm{d} t+\int_{Q} \chi \operatorname{div}\left(v \psi_{d+1}\right) \mathrm{d}(x, t) \\
& =\int_{0}^{\infty} \int \psi^{\prime}(x, t) \mathrm{d} \nabla \chi(t) \mathrm{d} t-\int_{0}^{\infty} \int v \psi_{d+1} \mathrm{~d} \nabla \chi(t) \mathrm{d} t
\end{aligned}
$$

for all $\psi=\left(\psi^{\prime}, \psi_{d+1}\right) \in C_{0}^{1}\left(Q ; \mathbb{R}^{d+1}\right)$ where $\psi_{d+1}(x, t) \in \mathbb{R}$. Moreover, since $q>d$, $L^{q}\left(0, T ; V_{q}\left(\mathbb{R}^{d}\right)\right) \cap L^{\infty}\left(0, T ; L_{\sigma}^{2}\left(\mathbb{R}^{d}\right)\right) \hookrightarrow L^{q}\left(0, T ; C_{0}\left(\mathbb{R}^{d}\right)\right)$ for each $T>0$ and

$$
\begin{aligned}
\left|\int_{0}^{\infty} \int \psi^{\prime}(x, t) \mathrm{d} \nabla \chi(t) \mathrm{d} t\right| & \leqslant C\left(E_{0}, T\right)\|\psi\|_{C_{0}\left(Q_{T}\right)}, \\
\left|\int_{0}^{\infty} \int v \psi_{d+1} \mathrm{~d} \nabla \chi(t) \mathrm{d} t\right| & \leqslant C\left(E_{0}, T\right)\|v\|_{L^{q}\left(0, T ; C_{0}\left(\mathbb{R}^{d}\right)\right)}\|\psi\|_{C_{0}\left(Q_{T}\right)} \\
& \leqslant C\left(E_{0}, T\right)\|\psi\|_{C_{0}\left(Q_{T}\right)}
\end{aligned}
$$

if supp $\psi \subseteq Q_{T}$ for $T>0$, where $E_{0}=\frac{1}{2}\left\|v_{0}\right\|_{2}^{2}+\kappa\left\|\nabla \chi_{0}\right\|_{\mathcal{M}}$. This shows that $\chi \in B V\left(Q_{T}\right)$ for every $0<T<\infty$.

\section{Approximate two-phase flow}

In the following we set $X_{\kappa}=B V$ if $\kappa>0$ and $X_{\kappa}=L^{\infty}$ if $\kappa=0$.

In order to formulate the approximation equations, let $\psi \in C_{0}^{\infty}\left(\mathbb{R}^{d}\right)$ with supp $\psi \subseteq \overline{B_{1}(0)}$, $\int \psi \mathrm{d} x=1$ and $\psi \geqslant 0$. Moreover, let $\Psi_{\varepsilon} f=\psi_{\varepsilon} * f$ if $\Omega=\mathbb{R}^{d}$ and $\Psi_{\varepsilon}=P_{\sigma}\left(\psi_{\varepsilon} * f\right)$, where $\psi_{\varepsilon}(x):=\varepsilon^{-d} \psi\left(\varepsilon^{-1} x\right), \varepsilon>0, f$ is extended by 0 to $\mathbb{R}^{d}$, and $P_{\sigma}$ denotes the Helmholtz projection (cf. [25]). Then we consider the approximate two-phase flow on $(0, T), T>0$, which is $v_{\varepsilon} \in L^{\infty}\left(0, T ; L_{\sigma}^{2}(\Omega)\right) \cap L^{q}\left(0, T ; V_{q}(\Omega)\right)$ that solves

$$
\begin{array}{r}
-\left(v_{\varepsilon}, \partial_{t} \varphi\right)_{Q_{T}}-\left(v_{0}, \varphi(0)\right)_{\Omega}-\left(\Psi_{\varepsilon} v_{\varepsilon} \otimes \psi_{\varepsilon} * v_{\varepsilon}, \nabla \psi_{\varepsilon} * \varphi\right)_{Q_{T}}+\left(S\left(\chi_{\varepsilon}, D v_{\varepsilon}\right), D \varphi\right)_{Q_{T}} \\
=\kappa \int_{0}^{T}\left\langle H_{\chi_{\varepsilon}(t)}, \Psi_{\varepsilon} \varphi(t)\right\rangle \mathrm{d} t
\end{array}
$$

for all $\varphi \in C_{(0)}^{\infty}(\Omega \times[0, T))^{d}$ with $\operatorname{div} \varphi=0$, and $\chi_{\varepsilon} \in L^{\infty}\left(0, T ; X_{K}(\Omega ;\{0,1\})\right)$ is the unique renormalized solution of the transport equation

$$
\begin{array}{rlrl}
\partial_{t} \chi_{\varepsilon}+\left(\Psi_{\varepsilon} v_{\varepsilon}\right) \cdot \nabla \chi_{\varepsilon} & =0 & & \text { in } Q_{T}, \\
\left.\chi_{\varepsilon}\right|_{t=0}=\chi_{0} & & \text { in } \Omega .
\end{array}
$$


Throughout this section we will frequently use the fact that

$$
\left(\Psi_{\varepsilon} v \otimes \psi_{\varepsilon} * w, \nabla \psi_{\varepsilon} * w\right)_{\Omega}=-\left(\Psi_{\varepsilon} v \otimes \psi_{\varepsilon} * w, \nabla \psi_{\varepsilon} * w\right)_{\Omega}=0
$$

for all $v, w \in L^{2}(\Omega)^{d}$, $\operatorname{div} v=0$. This follows from integration by parts and from $\operatorname{div} \Psi_{\varepsilon} v=0$, $\left.n \cdot \Psi_{\varepsilon}\right|_{\partial \Omega}=0$.

First of all, we need

Lemma 4.1 Let Assumption 1.1 hold and let $T, \varepsilon>0$. Then for every $v_{0} \in L_{\sigma}^{2}(\Omega)$, $f \in L^{q^{\prime}}\left(0, T ; V_{q}(\Omega)^{\prime}\right)$, and $\chi \in L^{\infty}\left(Q_{T} ;[0,1]\right)$, there is a unique $v \in L^{\infty}\left(0, T ; L_{\sigma}^{2}(\Omega)\right) \cap$ $L^{q}\left(0, T ; V_{q}(\Omega)\right)$ with $\partial_{t} v \in L^{q^{\prime}}\left(0, T ; V_{q}(\Omega)^{\prime}\right)$ solving

$$
-\left(v, \partial_{t} \varphi\right)_{Q}-\left(v_{0}, \varphi(0)\right)_{\Omega}-\left(\Psi_{\varepsilon} v \otimes \psi_{\varepsilon} * v, \nabla \psi_{\varepsilon} * v\right)_{Q}+(S(\chi, D v), D \varphi)_{Q}=\langle f, \varphi\rangle
$$

for all $\varphi \in C_{(0)}^{\infty}(\Omega \times[0, T))^{d}$ with $\operatorname{div} \varphi=0$. Moreover,

$$
\begin{array}{r}
\sup _{0 \leqslant t \leqslant T}\|v(t)\|_{2}^{2}+\|v\|_{L^{q}\left(0, T ; V_{q}\right)}^{q} \leqslant C\left(\|f\|_{L^{q^{\prime}\left(0, T ; V_{q}^{\prime}\right)}}^{q^{\prime}}+\left\|v_{0}\right\|_{2}^{2}\right), \\
\left\|\partial_{t} v\right\|_{L^{q^{\prime}\left(0, T ; V_{q}^{\prime}\right)}} \leqslant M\left(\|f\|_{L^{q^{\prime}\left(0, T ; V_{q}^{\prime}\right)}},\left\|v_{0}\right\|_{2}^{2}\right)
\end{array}
$$

for some continuous function $M$. Finally, if $f_{k}, f \in L^{1}\left(0, T ; L_{\sigma}^{2}(\Omega)\right) \cap L^{q^{\prime}}\left(0, T ; V_{q}(\Omega)^{\prime}\right)$ and $\chi_{k}, \chi \in L^{\infty}\left(Q_{T} ;[0,1]\right), k \in \mathbb{N}$, are bounded sequences such that $f_{k} \rightarrow f$ in $L^{1}\left(0, T ; L^{2}(\Omega)\right)$ and $\chi_{k} \rightarrow \chi$ in $L^{p}\left(Q_{T}\right)$ for some $1 \leqslant p \leqslant \infty$, then $v_{k} \rightarrow v \in C\left([0, T] ; L^{2}(\Omega)\right)$ where $v_{k}$ is the solution of 4.5 with $(f, \chi)$ replaced by $\left(f_{k}, \chi_{k}\right)$.

Proof. The proof of existence of solutions can be done by a standard Galerkin approximation using the fact that

$$
\langle A(u), v\rangle:=\int_{\Omega} v(\chi,|D u|) D u: D v \mathrm{~d} x, \quad u, v \in V:=V_{q}(\Omega),
$$

defines a strictly monotone, coercive, hemicontinuous bounded operator $A: V \rightarrow V^{\prime}$. More precisely:

First assume that the convective term is not present, i.e., $\Psi_{\varepsilon} \equiv 0$. If $\Omega$ is a bounded domain, then the lemma is a consequence of Zeidler [36, Theorem 30.A] with $V$ as above and $H=L^{2}(\Omega)$. The conditions (H1)-(H6) there are easily verified. If $\Omega=\mathbb{R}^{d}$, then $V=V_{q}\left(\mathbb{R}^{d}\right), H=L^{2}\left(\mathbb{R}^{d}\right)$, $V^{\prime}=V_{q}\left(\mathbb{R}^{d}\right)^{\prime}$ is no longer an evolution triple. But $V, H, V^{\prime}$ still have a common dense basis, and the fundamental relation

$$
(u(t), v(t))_{L^{2}(\Omega)}-(u(0), v(0))_{L^{2}(\Omega)}=\int_{0}^{t}\left(\left\langle u^{\prime}(s), v(s)\right\rangle+\left\langle v^{\prime}(s), u(s)\right\rangle\right) \mathrm{d} s
$$

still holds for all $0 \leqslant t \leqslant T$ and $u, v \in L^{q}(0, T ; V)$ with $u^{\prime}, v^{\prime} \in L^{q^{\prime}}\left(0, T ; V^{\prime}\right)$. Then the proof of [36. Theorem 30.A] easily carries over.

If the convective term is present, the proof can be easily modified using the fact that

$$
\left(\Psi_{\varepsilon} v \otimes \psi_{\varepsilon} * v, \nabla \psi_{\varepsilon} * v\right)_{Q}=0
$$

due to (4.4). Therefore the energy estimate for the case with convective term is the same as without it. Moreover, in order to pass to the limit in the convective term during the Galerkin approximation, one simply uses the fact that

$$
X:=\left\{u \in L^{q}\left(0, T ; V_{q}(\Omega)\right): \partial_{t} u \in L^{q^{\prime}}\left(0, T ; V_{q}(\Omega)^{\prime}\right)\right\} \hookrightarrow \hookrightarrow L^{1}\left(0, T ; L_{\mathrm{loc}}^{2}(\bar{\Omega})\right)
$$


because of 2.1 applied to $X_{0}=W_{q}^{1}\left(\Omega_{R}\right), X_{1}=L^{2}\left(\Omega_{R}\right)$, and $X_{2}=W_{q^{\prime}}^{-1}\left(\Omega_{R}\right)$, where $\Omega_{R}=$ $\Omega \cap B_{R}(0)$ and $R>0$ is arbitrary. This is sufficient to show that

$$
\lim _{n \rightarrow \infty}\left(\Psi_{\varepsilon} v_{n} \otimes \psi_{\varepsilon} * v_{n}, \nabla \psi_{\varepsilon} * \varphi\right)_{Q_{T}}=\left(\Psi_{\varepsilon} v \otimes \psi_{\varepsilon} * v, \nabla \psi_{\varepsilon} * \varphi\right)_{Q_{T}}
$$

for all $\varphi \in C_{(0)}^{\infty}(\Omega \times[0, T))^{d}$ with $\operatorname{div} \varphi=0$ if $v_{n} \rightarrow v$ in $X$.

Furthermore, we note that the estimate (4.6) follows from the usual energy estimate. In order to estimate $\partial_{t} v$, we observe that

$$
\|S(\chi, D v)\|_{L^{q^{\prime}}\left(Q_{T}\right)}^{q^{\prime}}=\int_{Q_{T}}|S(\chi, D v)|^{q /(q-1)} \mathrm{d}(x, t) \leqslant C \int_{Q_{T}}|D v|^{q} \mathrm{~d}(x, t) .
$$

Moreover, since $\Psi_{\varepsilon} u=P_{\sigma}\left(\psi_{\varepsilon} * u\right)$ and since $P_{\sigma}$ is continuous on $L^{s}(\Omega)^{d}$ for all $1<s<\infty$, we conclude that

$$
\left\|\Psi_{\varepsilon} v\right\|_{s} \leqslant C_{s}\left\|\psi_{\varepsilon} v\right\|_{s} \leqslant C_{\varepsilon, s}\|v\|_{2}, \quad\left\|\nabla \psi_{\varepsilon} * \varphi\right\|_{q} \leqslant C_{\varepsilon, q}\|\varphi\|_{V_{q}(\Omega)}
$$

for all $2 \leqslant s<\infty$. Therefore

$$
\begin{aligned}
\left|\int_{Q_{T}} \Psi_{\varepsilon} v \otimes \psi_{\varepsilon} * v: \nabla \psi_{\varepsilon} * \varphi \mathrm{d}(x, t)\right| & \leqslant C_{S} T^{1 / q^{\prime}}\left(\sup _{t \in[0, T]}\left\|\psi_{\varepsilon} * v(t)\right\|_{s}^{2}\right)\left\|\nabla \psi_{\varepsilon} * \varphi\right\|_{L^{q}\left(Q_{T}\right)} \\
& \leqslant C_{\varepsilon, s, T}\|v\|_{L^{\infty}\left(0, T ; L_{\sigma}^{2}(\Omega)\right)}^{2}\|\varphi\|_{L^{q}\left(0, T ; V_{q}(\Omega)\right)},
\end{aligned}
$$

where $1 / s=1 / 2-1 / 2 q$. Using these estimates and the equation (4.5), one easily derives (4.7).

In order to prove uniqueness and the last statement, let $v, w$ be two solutions of 4.5 . Then

$$
\begin{aligned}
& -\left(v-w, \partial_{t} \varphi\right) Q_{T}+(S(\chi, D v)-S(\chi, D w), D \varphi)_{Q_{T}} \\
& \quad=\left(\Psi_{\varepsilon}(v-w) \otimes \psi_{\varepsilon} * v, \nabla \psi_{\varepsilon} * \varphi\right)_{Q_{T}}+\left(\Psi_{\varepsilon} w \otimes \psi_{\varepsilon} *(v-w), \nabla \psi_{\varepsilon} * \varphi\right)_{Q_{T}}
\end{aligned}
$$

for all $\varphi \in C_{(0)}^{\infty}(\Omega \times[0, T))^{d}$ with $\operatorname{div} \varphi=0$. Choosing $\varphi=(w-v) \chi_{[0, t]}, t \in[0, T]$, via a standard approximation and using $(4.8)$ we conclude that

$$
\begin{aligned}
\|v(t)-w(t)\|_{2}^{2} & \leqslant\|v(t)-w(t)\|_{2}^{2}+(S(\chi, D v)-S(\chi, D w), D v-D w) Q_{t} \\
& \leqslant C_{\varepsilon}\left(\sup _{0 \leqslant t \leqslant T}\left(\|v(t)\|_{2}+\|w(t)\|_{2}\right)\right) \int_{0}^{t}\|v(s)-w(s)\|_{2}^{2} \mathrm{~d} s
\end{aligned}
$$

since $D v \mapsto S(\chi, D v)$ is monotone. Hence Gronwall's inequality implies $v \equiv w$.

Finally, let $f_{k}, f, \chi_{k}, \chi, v_{k}, v$ be as in the last statement. Then

$$
\begin{aligned}
-\left(v_{k}-v, \partial_{t} \varphi\right)_{Q}+\left(S\left(\chi_{k}, D v_{k}\right)-S\left(\chi_{k}, D v\right), D \varphi\right)_{Q} \\
=\left(S(\chi, D v)-S\left(\chi_{k}, D v\right), D \varphi\right)_{Q}+\left\langle f_{k}-f, \varphi\right\rangle \\
\quad+\left(\Psi_{\varepsilon}\left(v_{k}-v\right) \otimes \psi_{\varepsilon} * v_{k}, \nabla \psi_{\varepsilon} * \varphi\right)_{Q_{T}}+\left(\Psi_{\varepsilon} v \otimes \psi_{\varepsilon} *\left(v_{k}-v\right), \nabla \psi_{\varepsilon} * \varphi\right)_{Q_{T}}
\end{aligned}
$$

for all $\varphi \in C_{(0)}^{\infty}(\Omega \times[0, T))^{d}$ with $\operatorname{div} \varphi=0$. Choosing $\varphi=\left(v_{k}-v\right) \chi_{[0, t]}, t \in[0, T]$, we conclude, using the boundedness of $v_{k}, v$, that 


$$
\begin{aligned}
& \left\|v_{k}(t)-v(t)\right\|_{2}^{2}+\left(S\left(\chi_{k}, D v_{k}\right)-S\left(\chi_{k}, D v\right), D v_{k}-D v\right)_{Q_{t}} \\
& \leqslant C_{\varepsilon}\left(\left\|f_{k}-f\right\|_{L^{1}\left(0, T ; L^{2}\right)}+\left\|S(\chi, D v)-S\left(\chi_{k}, D v\right)\right\|_{L^{q^{\prime}}\left(Q_{T}\right)}+\int_{0}^{t}\left\|v_{k}(s)-v(s)\right\|_{2}^{2} \mathrm{~d} s\right) .
\end{aligned}
$$

Thus

$$
\sup _{0 \leqslant t \leqslant T}\left\|v_{k}(t)-v(t)\right\|_{2}^{2} \leqslant C_{\varepsilon, T}\left(\left\|f_{k}-f\right\|_{L^{1}\left(0, T ; L^{2}\right)}+\left\|S(\chi, D v)-S\left(\chi_{k}, D v\right)\right\|_{L^{q^{\prime}}\left(Q_{T}\right)}\right)
$$

by Gronwall's inequality. The second term can be estimated as

$$
\begin{aligned}
& \left\|S(\chi, D v)-S\left(\chi_{k}, D v\right)\right\|_{L^{q^{\prime}}\left(Q_{T}\right)}^{q^{\prime}} \leqslant C_{0} \int_{Q_{T}}\left|\chi-\chi_{k}\right||D v|^{q} \mathrm{~d}(x, t) \\
& \quad \leqslant C_{1} \int_{Q_{T}}\left|\chi-\chi_{k}\right||D \varphi|^{q} \mathrm{~d}(x, t)+C_{2} \int_{Q_{T}}|D v-D \varphi|^{q} \mathrm{~d}(x, t)
\end{aligned}
$$

for all $\varphi \in C_{(0)}^{\infty}\left(\bar{Q}_{T}\right)$. Now we observe that the first term on the right-hand side converges to zero as $k \rightarrow \infty$ since $\chi_{k} \rightarrow \chi$ in $L^{p}\left(Q_{T}\right)$, and the second term is arbitrarily small since $C_{(0)}^{\infty}\left(\bar{Q}_{T}\right)$ is dense in $L^{q}\left(0, T ; \dot{W}_{q}^{1}(\Omega)\right)$. Hence $\lim _{k \rightarrow \infty}\left\|S(\chi, D v)-S\left(\chi_{k}, D v\right)\right\|_{L^{q^{\prime}}\left(Q_{T}\right)}=0$. Altogether this implies $\lim _{k \rightarrow \infty} \sup _{0 \leqslant t \leqslant T}\left\|v_{k}(t)-v(t)\right\|_{2}^{2}=0$.

THEOREM 4.2 Let Assumption 1.1 hold. Then for every $\varepsilon, T>0, v_{0} \in L_{\sigma}^{2}(\Omega), \chi_{0} \in$ $L^{\infty}(\Omega ;\{0,1\})$ if $\kappa=0$, and $\chi_{0}=\chi_{\Omega_{0}^{+}}$if $\kappa>0$, where $\Omega_{0}^{+} \Subset \Omega$ is a bounded domain with $C^{1}$-boundary, there is a solution $v_{\varepsilon} \in L^{\infty}\left(0, T ; L_{\sigma}^{2}(\Omega)\right) \cap L^{q}\left(0, T ; V_{q}(\Omega)\right), \chi_{\varepsilon} \in$ $L^{\infty}\left(0, T ; X_{\kappa}(\Omega ;\{0,1\})\right)$ of 4.1$]-4.3$. Moreover, every solution satisfies the energy equality

$$
\frac{1}{2}\left\|v_{\varepsilon}(t)\right\|_{2}^{2}+\kappa\left\|\nabla \chi_{\varepsilon}(t)\right\|_{\mathcal{M}}+\int_{s}^{t} \int_{\Omega} S\left(\chi_{\varepsilon}, D v_{\varepsilon}\right): D v_{\varepsilon} \mathrm{d} x \mathrm{~d} \tau=\frac{1}{2}\left\|v_{\varepsilon}(s)\right\|_{2}^{2}+\kappa\left\|\nabla \chi_{\varepsilon}(s)\right\|_{\mathcal{M}}
$$

for all $s, t \in(0, T), s \leqslant t$, where $t \mapsto \frac{1}{2}\left\|v_{\varepsilon}(t)\right\|_{2}^{2}$ and $t \mapsto\left\|\nabla \chi_{\varepsilon}(t)\right\|_{\mathcal{M}}$ are absolutely continuous functions satisfying

$$
\frac{\mathrm{d}}{\mathrm{d} t} \frac{1}{2}\left\|v_{\varepsilon}(t)\right\|_{2}^{2}=\left\langle\partial_{t} v_{\varepsilon}(t), v_{\varepsilon}(t)\right\rangle_{\Omega}, \quad \frac{\mathrm{d}}{\mathrm{d} t}\left\|\nabla \chi_{\varepsilon}(t)\right\|_{\mathcal{M}}=-\left\langle H_{\chi_{\varepsilon}(t)}, \Psi_{\varepsilon} * v_{\varepsilon}(t)\right\rangle \quad \text { if } \kappa>0
$$

for almost all $t \in(0, T)$ and $\partial_{t} v_{\varepsilon}, \kappa\left\langle H_{\chi_{\varepsilon}(t)}, \psi_{\varepsilon} * \cdot\right\rangle \in L^{q^{\prime}}\left(0, T ; V_{q}(\Omega)^{\prime}\right)$.

Proof. Let

$$
X_{1}:=\left\{u \in L^{q}\left(0, T ; V_{q}(\Omega)\right): \partial_{t} u \in L^{q^{\prime}}\left(0, T ; V_{q}(\Omega)^{\prime}\right)\right\}
$$

normed in a suitable way. Moreover, let $X_{0}:=C\left([0, T] ; L_{\sigma}^{2}(\Omega)\right)$ and let $X_{\alpha}=\left(X_{0}, X_{1}\right)_{\alpha}$, where $(\cdot, \cdot)_{\alpha}$ is an exact interpolation functor of type $\alpha \in(0,1)$ (e.g. the real interpolation functor, cf. Bergh and Löfström [5]). Note that by [4.8), $X_{1} \hookrightarrow X_{0}$. Furthermore, we note that the inclusion of $X_{1}$ into $L^{1}\left(0, T ; L_{\text {loc }}^{2}(\bar{\Omega})\right)$ is compact because of 2.1$)$ applied to $W_{q}^{1}\left(\Omega_{R}\right), L^{2}\left(\Omega_{R}\right)$, and $W_{q^{\prime}}^{-1}\left(\Omega_{R}\right)$, where $\Omega_{R}=\Omega \cap B_{R}(0)$ and $R>0$ is arbitrary. By [5, Theorem 3.8.1] the same holds for $X_{\alpha}, \alpha \in(0,1]$, in place $X_{1}$. 
We define a mapping $F: X_{0} \rightarrow X_{1}$ as follows: For given $u \in X_{0}$ let $\chi_{u} \in$ $L^{\infty}\left(0, \infty ; X_{\kappa}(\Omega ;\{0,1\})\right)$ be the solution of the transport equation $2.9-(2.10)$ with $v$ in 2.9 ) replaced by $\Psi_{\varepsilon} u$. Then

$$
X_{0} \ni u \mapsto \chi_{u} \in L^{p}\left(Q_{T}\right), \quad 1<p<\infty,
$$

is strongly continuous by Lemma 2.5 Moreover, the mapping $X_{\alpha} \ni u \mapsto \chi_{u} \in L^{p}\left(Q_{T}\right), \alpha \in(0,1]$, is even compact by the following argument: If $u_{k} \in X_{\alpha}, k \in \mathbb{N}_{0}$, is a bounded sequence, then after passing to a suitable subsequence, $u_{k} \rightarrow u$ in $L^{1}\left(0, T ; L_{\text {loc }}^{2}(\bar{\Omega})\right)$ by the observations above. This implies the same statement for $\Psi_{\varepsilon} u_{k}, \Psi_{\varepsilon} u$. Hence $\chi_{u_{k}} \rightarrow \chi_{u}$ again by Lemma 2.5 .

Now let $v=F(u)$ be the solution of 4.5 with $\chi=\chi_{u}$ and

$$
\left\langle f_{u}, \varphi\right\rangle:=\kappa \int_{0}^{T}\left\langle H_{\chi_{u}(t)}, \Psi_{\varepsilon} \varphi(t)\right\rangle \mathrm{d} t
$$

Claim. $\quad F: X_{0} \rightarrow X_{0}$ is continuous, $F: X_{\alpha} \rightarrow X_{0}, \alpha \in(0,1]$, is compact, and $F: X_{0} \rightarrow X_{1}$ is bounded.

Proof of Claim. First let $\kappa=0$. Then $F: X_{0} \rightarrow X_{0}$ is continuous because of Lemma 4.1 and 4.10). Moreover, $F: X_{0} \rightarrow X_{1}$ is bounded by (4.6) and 4.7). Finally, $F: X_{1} \rightarrow X_{0}$ is compact since $X_{1} \ni u \mapsto \chi_{0} \in L^{p}\left(Q_{T}\right)$ is compact and the mapping of $\chi_{u}$ to the solution $v=F(u) \in X_{0}$ of (4.5) with $\chi=\chi_{u}$ and $f=0$ is continuous.

In the case $\kappa>0$ it remains to prove that $X_{0} \ni u \mapsto f_{u} \in L^{q^{\prime}}\left(0, T ; V_{q}\left(\mathbb{R}^{d}\right)^{\prime}\right)$ is bounded, that $X_{0} \ni u \mapsto f_{u} \in L^{1}\left(0, T ; L_{\sigma}^{2}\left(\mathbb{R}^{d}\right)\right)$ is continuous, and that $X_{\alpha} \ni u \mapsto f_{u} \in L^{1}\left(0, T ; L_{\sigma}^{2}\left(\mathbb{R}^{d}\right)\right)$, $\alpha \in(0,1]$, is compact. Then the claim follows in the same way.

Firstly, we estimate $f_{u}$. Since $\Omega=\mathbb{R}^{d}$ if $\kappa>0, \Psi_{\varepsilon} \varphi=\psi_{\varepsilon} * \varphi$ and

$$
\left|\left\langle H_{\chi_{u}(t)}, \Psi_{\varepsilon} \varphi(t)\right\rangle\right| \leqslant C \kappa\left\|\nabla \chi_{u}(t)\right\| \mathcal{M}\left\|\nabla \psi_{\varepsilon} * \varphi\right\|_{C_{b}^{0}\left(\mathbb{R}^{d}\right)} \leqslant C_{\varepsilon} \kappa\left\|\nabla \chi_{u}(t)\right\| \mathcal{M}\|\varphi\|_{Y},
$$

where $Y=L_{\sigma}^{2}\left(\mathbb{R}^{d}\right)$ or $Y=V_{q}\left(\mathbb{R}^{d}\right)$ and

$$
\begin{aligned}
\left\|\nabla \chi_{u}\right\|_{L^{\infty}\left(0, T ; \mathcal{M}\left(\mathbb{R}^{d}\right)\right)} & \leqslant M\left(\left\|\Psi_{\varepsilon} u\right\|_{C\left([0, T] ; C_{b}^{2}\left(\mathbb{R}^{d}\right)\right)}\right)\left\|\chi_{0}\right\|_{B V\left(\mathbb{R}^{d}\right)} \\
& \leqslant M^{\prime}\left(\varepsilon,\|u\|_{C\left([0, T] ; L_{\sigma}^{2}\left(\mathbb{R}^{d}\right)\right)}\right)\left\|\chi_{0}\right\|_{B V\left(\mathbb{R}^{d}\right)}
\end{aligned}
$$

by 2.12 . Hence

$$
\left\|f_{u}\right\|_{L^{q^{\prime}}\left(0, T ; V_{q}^{\prime}\right)}+\left\|f_{u}\right\|_{L^{1}\left(0, T ; L_{\sigma}^{2}\right)} \leqslant M\left(\varepsilon, T,\|u\|_{C\left([0, T] ; L_{\sigma}^{2}\left(\mathbb{R}^{d}\right)\right)}\right) \kappa\left\|\chi_{0}\right\|_{B V\left(\mathbb{R}^{d}\right)} .
$$

In particular this implies

$$
\|F(u)\|_{X_{1}} \leqslant M\left(\varepsilon, T,\left\|v_{0}\right\|_{2},\|u\|_{L^{\infty}\left(0, T ; L^{2}(\Omega)\right)}, \kappa\left\|\nabla \chi_{u}\right\|_{L^{\infty}(0, T ; \mathcal{M}(\Omega))}\right)
$$

for another continuous function $M$ and $\kappa \geqslant 0$ by 4.6) and 4.7).

Now let $u_{k} \in X_{\alpha}, k \in \mathbb{N}$, be a bounded sequence and let $\alpha \in(0,1]$. If $\alpha=1$, then $\Psi_{\varepsilon} u_{k} \in$ $C\left([0, T] ; C^{1}\left(\overline{B_{R}(0)}\right)\right), k \in \mathbb{N}$, is precompact for any $R>0$ since $\Psi_{\varepsilon} u_{k} \in C\left([0, T] ; C_{b}^{2}\left(\mathbb{R}^{d}\right)\right)$ and $\partial_{t} \Psi_{\varepsilon} u_{k}=\Psi_{\varepsilon} \partial_{t} u_{k} \in L^{q^{\prime}}\left(0, T ; C_{b}^{2}\left(\mathbb{R}^{d}\right)\right)$ are uniformly bounded. Now using again [5], Theorem 3.8.1] we conclude that $\Psi_{\varepsilon} u_{k} \in C\left([0, T] ; C^{1}\left(\overline{B_{R}(0)}\right)\right), k \in \mathbb{N}$, is precompact if $\alpha \in(0,1)$. 
Therefore for a suitable subsequence, $\Psi_{\varepsilon} u_{k} \rightarrow \Psi_{\varepsilon} u$ in $C\left([0, T] ; C^{1}\left(\overline{B_{R}(0)}\right)\right)$ for any $R>0$ as $k \rightarrow \infty$. Hence 2.16 implies that

$$
\lim _{k \rightarrow \infty}\left\langle H_{\chi_{u_{k}}(t)}, \varphi\right\rangle=\left\langle H_{\chi_{u}(t)}, \varphi\right\rangle \quad \text { for all } \varphi \in C_{0}^{1}\left(\mathbb{R}^{d}\right)^{d}
$$

uniformly in $t \in[0, T]$. Moreover, since supp $\chi_{u_{k}}, k \in \mathbb{N}$, is contained in a compact set $K$ by Lemma 2.4 and $C^{2}(K) \hookrightarrow \hookrightarrow C^{1}(K)$,

$$
\lim _{k \rightarrow \infty} \sup _{t \in[0, T]\|\varphi\|_{C_{b}^{2}\left(\mathbb{R}^{d}\right)} \leqslant 1}\left|\left\langle H_{\chi_{u_{k}}(t)}, \varphi\right\rangle-\left\langle H_{\chi_{u}(t)}, \varphi\right\rangle\right|=0 .
$$

Therefore $f_{u_{k}} \rightarrow f_{u} \in L^{1}\left(0, T ; L_{\sigma}^{2}\left(\mathbb{R}^{d}\right)\right)$ since $\Psi_{\varepsilon}: L^{2}\left(\mathbb{R}^{d}\right) \rightarrow C_{b}^{2}\left(\mathbb{R}^{d}\right)$. By the same arguments it follows that $f_{u} \in L^{1}\left(0, T ; L_{\sigma}^{2}\left(\mathbb{R}^{d}\right)\right)$ depends continuously on $u \in X_{0}$. This finishes the proof of the claim.

Now, since $F: X_{\alpha} \rightarrow X_{1}$ is bounded and $F: X_{\alpha} \rightarrow X_{0}$ is continuous for all $\alpha \in[0,1]$, the interpolation inequality $\|u\|_{X_{\alpha}} \leqslant\|u\|_{X_{0}}^{1-\alpha}\|u\|_{X_{1}}^{\alpha}$ implies that $F: X_{\alpha} \rightarrow X_{\alpha}$ is continuous for all $\alpha \in[0,1)$. Similarly, the boundedness of $F: X_{\alpha} \rightarrow X_{1}, \alpha \in[0,1]$, and the compactness of $F: X_{\alpha} \rightarrow X_{0}, \alpha \in(0,1]$, yields the compactness of $F: X_{\alpha} \rightarrow X_{\alpha}, \alpha \in(0,1]$. Altogether $F: X_{\alpha} \rightarrow X_{\alpha}$ is a completely continuous mapping for all $\alpha \in(0,1)$.

In order to prove the existence of a fixed point $v_{\varepsilon}=F\left(v_{\varepsilon}\right) \in X_{\alpha}, \alpha \in(0,1)$, we will use the Leray-Schauder principle (cf. e.g. Sohr [28, Lemma 3.1.1, Chapter II]), for which it only remains to verify the following condition for a suitable $R>0$ :

$$
\text { If } v=\lambda F(v) \text { for some } v \in X_{\alpha}, \lambda \in[0,1] \text {, then }\|v\|_{X_{\alpha}} \leqslant R \text {. }
$$

Therefore we assume that $v=\lambda F(v)$ for some $v \in X_{\alpha}, \lambda \in[0,1], \alpha \in(0,1)$. If $\lambda=0$, then obviously, $\|v\|_{X_{\alpha}}=0 \leqslant R$ for any $R>0$. Thus it remains to consider the case $\lambda>0$. Set $\alpha=\lambda^{-1} \geqslant 1$. Then $u=\alpha v=F(v)$ solves

$$
\begin{aligned}
-\left(u, \partial_{t} \varphi\right)_{Q_{T}}-\left(v_{0}, \varphi(0)\right)_{\Omega}-\left(\Psi_{\varepsilon} u \otimes \psi_{\varepsilon} * u, \nabla \psi_{\varepsilon} * \varphi\right)_{Q_{T}}+ & \left(S\left(\chi_{v}, D u\right), D \varphi\right)_{Q_{T}} \\
& =\kappa \int_{0}^{T}\left\langle H_{\chi_{v}}(t), \Psi_{\varepsilon} \varphi(t)\right\rangle \mathrm{d} t
\end{aligned}
$$

for all $\varphi \in C_{(0)}^{\infty}(\Omega \times[0, T))^{d}$ with $\operatorname{div} \varphi=0$. Hence choosing $\varphi=u \chi_{[0, t]}$ (after a standard approximation) we conclude that

$$
\frac{1}{2}\|u(t)\|_{2}^{2}+\int_{0}^{t} \int_{\Omega} S\left(\chi_{v}, D u\right): D u \mathrm{~d} x \mathrm{~d} \tau=\frac{1}{2}\left\|v_{0}\right\|_{2}^{2}+\kappa \int_{0}^{t}\left\langle H_{\chi_{v}(\tau)}, \Psi_{\varepsilon} u(\tau)\right\rangle \mathrm{d} \tau .
$$

where we have used (4.4).

Now, since $\Psi_{\varepsilon} u(\tau)=\alpha \Psi_{\varepsilon} v(\tau)$, Lemma2.3 implies that

$$
\int_{0}^{t}\left\langle H_{\chi_{v}(\tau)}, \Psi_{\varepsilon} u(\tau)\right\rangle \mathrm{d} \tau=\alpha\left(\left|\nabla \chi_{0}\right|(\Omega)-\left|\nabla \chi_{v}(t)\right|(\Omega)\right) \quad \text { if } \kappa>0 .
$$

Hence

$$
\frac{1}{2} \alpha\|v(t)\|_{2}^{2}+\kappa\left|\nabla \chi_{v}(t)\right|(\Omega)+\alpha^{q-1} c_{0} \int_{0}^{t} \int_{\Omega}|D v|^{q} \mathrm{~d} x \mathrm{~d} \tau \leqslant \frac{1}{2}\left\|v_{0}\right\|_{2}^{2}+\kappa\left|\nabla \chi_{0}\right|(\Omega)=: E_{0}
$$


for all $0 \leqslant t \leqslant T$, where $c_{0}$ is as in (1.12). Hence, using (4.11) and the last estimate, we conclude that

$$
\|v\|_{X_{\alpha}} \leqslant C \lambda\|F(v)\|_{X_{1}} \leqslant M^{\prime}\left(\varepsilon, T,\|v\|_{L^{\infty}\left(0, T ; L^{2}(\Omega)\right)}, \kappa\left\|\nabla \chi_{v}\right\|_{L^{\infty}(0, T ; \mathcal{M}(\Omega))}\right) \leqslant M^{\prime \prime}\left(\varepsilon, T, E_{0}\right)
$$

for some continuous functions $M^{\prime}, M^{\prime \prime}$. Hence for $R:=M^{\prime \prime}\left(\varepsilon, T, E_{0}\right)$ the condition 4.12) is valid. This implies that there is a fixed point $v_{\varepsilon}=F\left(v_{\varepsilon}\right) \in X_{\alpha}$, which is a solution of (4.1) 4.3 by definition of $F$.

The remaining statements easily follow from 4.8 and 2.13 .

\section{Proofs of the main theorems}

\subsection{Approximation sequence}

Throughout this section we assume that the assumptions of Theorem 1.6 if $\kappa>0$ and Theorem 1.9 if $\kappa=0$ hold. Moreover, we denote by $E_{0}=\frac{1}{2}\left\|v_{0}\right\|_{2}^{2}+\kappa\left|\nabla \chi_{0}\right|(\Omega)$ the initial energy of the flow.

For every $\varepsilon>0$ let $\left(v_{\varepsilon}, \chi_{\varepsilon}\right)$ be an approximate solution due to Theorem 4.2 for $T=1 / \varepsilon$. Because of the uniform bounds of $\left(v_{\varepsilon}, \chi_{\varepsilon}\right)$ given by the energy equality (4.9) and

$$
\left\|S\left(\chi_{k}, D v_{k}\right)\right\|_{L^{q^{\prime}}\left(Q_{T}\right)}^{q^{\prime}}=\int_{Q_{T}}\left|S\left(\chi_{k}, D v_{k}\right)\right|^{q /(q-1)} \mathrm{d}(x, t) \leqslant C \int_{Q_{T}}\left|D v_{k}\right|^{q} \mathrm{~d}(x, t)
$$

due to 1.12 there is a subsequence $\left(v_{\varepsilon_{k}}, \chi_{\varepsilon_{k}}\right) \equiv\left(v_{k}, \chi_{k}\right), k \in \mathbb{N}$, such that

$$
\begin{aligned}
v_{k} \rightarrow v & \text { in } L^{q}\left(0, \infty ; V_{q}(\Omega)\right), \\
v_{k} \rightarrow^{*} v & \text { in } L^{\infty}\left(0, \infty ; L_{\sigma}^{2}(\Omega)\right), \\
S\left(\chi_{k}, D v_{k}\right) \rightarrow \tilde{S} & \text { in } L^{q^{\prime}}(Q), \\
\chi_{k} \rightarrow^{*} \chi & \text { in } L^{\infty}(Q), \\
\nabla \chi_{k} \rightarrow^{*} \nabla \chi & \text { in } L^{\infty}\left(0, \infty ; H^{-s}(\Omega)\right), s>d / 2, \text { if } \kappa>0,
\end{aligned}
$$

for some $v \in L^{\infty}\left(0, \infty ; L_{\sigma}^{2}(\Omega)\right) \cap L^{q}\left(0, \infty ; V_{q}(\Omega)\right), \chi \in L^{\infty}\left(0, \infty ; X_{\kappa}(\Omega)\right)$ with $X_{\kappa}=B V$ if $\kappa>0$ and $X_{\kappa}=L^{\infty}$ if $\kappa=0$, and $\tilde{S} \in L^{q^{\prime}}(Q)$. Here the functions $v_{\varepsilon}, \chi_{\varepsilon}$ are extended by 0 for $t>1 / \varepsilon$.

\subsection{Passing to the limit in the transport equation and the convective term}

We pass to the limit in the transport equation using the following lemma, which is a variant of [18, Lemma 5.1]:

LEMmA 5.1 Let $\left(v_{k}, \chi_{k}\right)_{k \in \mathbb{N}}$ be bounded in $L_{\text {loc }}^{q}\left([0, \infty) ; W_{q}^{1}\left(\Omega ; \mathbb{R}^{d}\right)\right) \times L^{\infty}(Q), 1<q<\infty$, such that

$$
\begin{array}{ll}
v_{k} \rightarrow v & \text { in } L^{q}\left(0, T ; W_{q}^{1}(\Omega)^{d}\right) \text { for all } T>0, \\
\chi_{k} \rightarrow^{*} \chi & \text { in } L^{\infty}(Q) .
\end{array}
$$

If $\left(\partial_{t} \chi_{k}\right)_{k \in \mathbb{N}}$ is bounded in $L^{q^{\prime}}\left(0, T ; W_{q^{\prime}, \text { loc }}^{-1}(\bar{\Omega})\right)$ for any $T>0$, then $\chi_{k} v_{k} \rightarrow \chi v$ in $\mathcal{D}^{\prime}(Q)$. 
Proof. First of all, since the statement is local, it is sufficient to consider the case that $\Omega$ is a bounded domain and $(0, \infty)$ is replaced by $(0, T), T>0$ arbitrary. Because of $L^{q^{\prime}}(\Omega) \hookrightarrow \hookrightarrow W_{q^{\prime}}^{-1}(\Omega), 2.1$ yields

$$
\chi_{k_{j}} \rightarrow \chi^{*} \quad \text { in } L^{q^{\prime}}\left(0, T ; W_{q^{\prime}}^{-1}(\Omega)\right)
$$

for some subsequence. Since $\chi_{k} \rightarrow^{*} \chi$ in $L^{\infty}\left(Q_{T}\right)$, we have $\chi^{*}=\chi$ and the full sequence $\chi_{k}$ converges strongly in $L^{q^{\prime}}\left(0, T ; W_{q^{\prime}}^{-1}(\Omega)\right)$. This implies that $\chi_{k} v_{k} \rightarrow \chi v$ in $\mathcal{D}^{\prime}\left(Q_{T}\right)$.

COROLlary 5.2 Let $(v, \chi)$ be as in 5.1)- 5.5). Then $(v, \chi)$ solves the transport equation (1.10)(1.11).

Proof. It only remains to observe that $\partial_{t} \chi_{k}$ is bounded in $L^{q^{\prime}}\left(0, T ; W_{q^{\prime}, \text { loc }}^{-1}(\bar{\Omega})\right)$ for any $T>0$ : Because of

$$
\left(\chi_{k}, \partial_{t} \varphi\right)_{Q_{T}}+\left(\chi_{k}, v_{k} \cdot \nabla \varphi\right)_{Q_{T}}=0 \quad \text { for all } \varphi \in C_{0}^{\infty}\left(Q_{T}\right)
$$

we estimate

$$
\begin{aligned}
\left|\left(\chi_{k}, \partial_{t} \varphi\right)_{Q_{T}}\right| & \leqslant\left\|v_{k}\right\|_{L^{q}\left([0, T] \times \Omega_{R}\right)}\|\nabla \varphi\|_{L^{q^{\prime}}\left(Q_{T}\right)} \\
& \leqslant C\left(\left\|v_{k}\right\|_{L^{q}\left(0, T ; V_{q}(\Omega)\right)}+T^{1 / q}\left\|v_{k}\right\|_{L^{\infty}\left(0, T ; L^{2}(\Omega)\right)}\right)\|\nabla \varphi\|_{L^{q^{\prime}}\left(Q_{T}\right)}
\end{aligned}
$$

for all $\varphi \in L^{q^{\prime}}\left(0, T ; W_{q^{\prime}}^{1}(\Omega)\right)$ with $\operatorname{supp} \varphi \subseteq \bar{\Omega}_{R}, \Omega_{R}=\Omega \cap B_{R}, R>0$. Note that $V_{q}\left(\mathbb{R}^{d}\right) \cap$ $L_{\sigma}^{2}\left(\mathbb{R}^{d}\right) \hookrightarrow L^{q}\left(B_{R}(0)\right)$ if $\Omega=\mathbb{R}^{d}$ and $V_{q}(\Omega) \hookrightarrow L^{q}(\Omega)$ if $\Omega$ is bounded.

The last corollary and 2.11) yield

$$
\|\chi\|_{L^{p}\left(Q_{T}\right)}^{p}=\|\chi\|_{L^{1}\left(Q_{T}\right)}=T\left\|\chi_{0}\right\|_{L^{1}(\Omega)}=\left\|\chi_{k}\right\|_{L^{p}\left(Q_{T}\right)}^{p}
$$

for all $1 \leqslant p<\infty$ since $\int \chi(t) \mathrm{d} x=\int \chi_{0} \mathrm{~d} x$ for almost all $t>0$. Hence

$$
\chi_{k} \rightarrow \chi \quad \text { in } L^{p}\left(Q_{T}\right) \text { for all } 1 \leqslant p<\infty, T>0 .
$$

In particular this implies that $\chi(x, t) \in\{0,1\}$ almost everywhere.

In order to pass to the limit in the convective term, we use the following lemma.

LEMMA 5.3 Let $v_{k}, v$ be as above and let $q>2 d /(d+2)$. Then

$$
v_{k} \rightarrow v \quad \text { in } L^{q^{\prime}}\left(0, T ; L_{\mathrm{loc}}^{2}(\bar{\Omega})\right)
$$

for all $T>0$. In particular,

$$
\lim _{k \rightarrow \infty}\left(\Psi_{k} v_{k} \otimes \psi_{k} * v_{k}, \nabla \psi_{k} * \varphi\right)_{Q}=(v \otimes v, \nabla \varphi)_{Q}
$$

for all $\varphi \in C_{(0)}^{\infty}(\bar{Q})^{d}$.

Proof. First let $|\Omega|<\infty$. Since $S\left(\chi_{k}, D v_{k}\right)$ and $\Psi_{k} v_{k} \otimes \psi_{k} * v_{k}$ are uniformly bounded in $L^{q^{\prime}}\left(Q_{T}\right)$, $L^{\infty}\left(0, T ; L^{1}(\Omega)\right)$, resp., and

$$
\left|\int_{0}^{\infty}\left\langle H_{\chi_{k}(t)}, \varphi(t)\right\rangle \mathrm{d} t\right| \leqslant \sup _{0 \leqslant t<\infty}\left\|\nabla \chi_{k}(t)\right\|_{\mathcal{M}\left(\mathbb{R}^{d}\right)} \int_{0}^{\infty}\|\varphi(t)\|_{C_{b}^{1}\left(\mathbb{R}^{d}\right)} \mathrm{d} t \quad \text { if } \kappa>0,
$$


$\partial_{t} v_{k}$ is uniformly bounded in $L^{q^{\prime}}\left(0, T ; H^{-m}(\Omega)\right)$ for some suitable $m \in \mathbb{N}$. Using 2.1 with $V_{q}(\Omega) \cap L^{2}(\Omega) \hookrightarrow \hookrightarrow L^{2}(\Omega) \hookrightarrow H^{-m}(\Omega)$ when $q>2 d /(d+2)$ proves $[5.8)$ in the case $|\Omega|<\infty$. The case $\Omega=\mathbb{R}^{d}$ follows from the first part applied to $\Omega^{\prime} \Subset \mathbb{R}^{d}$.

Finally, 5.8 implies that $\psi_{k} * v_{k} \cdot \nabla \psi_{k} * \varphi \rightarrow v \cdot \nabla \varphi$ in $L^{q^{\prime}}\left(0, T ; L^{2}(\Omega)\right)$ for all $\varphi \in C_{(0)}^{\infty}(\bar{Q})^{d}$ and $T>0$ since $\psi_{k}$ converges strongly to the identity as $k \rightarrow \infty$. Together with (5.2) this implies the last statement.

\subsection{Case without surface tension}

Obviously, in the case of two Newtonian fluids, i.e., $q=2$ and $v(j, s)=v_{j}$ are constant, the strong convergence of $\chi_{k}$ and the weak convergence of $D v_{k}$ yield $\tilde{S}=S(\chi, D v)$. For the case $q \neq 2$ and $\kappa=0$, we use the following lemma:

LEMMA 5.4 Let $\kappa=0$ and let $q \geqslant 2 d /(d+2)+1$. Then

$$
S(\chi(x, t), D v(x, t))=\tilde{S}(x, t) \quad \text { for almost all }(x, t) \in Q .
$$

Proof. By the results so far we obtain

$$
-\left(v, \partial_{t} \varphi\right)_{Q}-\left(v_{0}, \varphi(0)\right)_{\Omega}-(v \otimes v, \nabla \varphi)_{Q}+(\tilde{S}, D \varphi)_{Q}=0
$$

for all $\varphi \in C_{(0)}^{\infty}([0, \infty) \times \Omega)^{d}$ with $\operatorname{div} \varphi=0$. Furthermore, $q \geqslant 2 d /(d+2)+1$ implies that for all $T>0$,

$$
\left|(v \otimes v, \nabla \varphi)_{Q_{T}}\right| \leqslant C\|\nabla \varphi\|_{L^{q}\left(Q_{T}\right)}
$$

for all $\varphi \in C_{(0)}^{\infty}([0, T) \times \Omega)^{d}$ with $\operatorname{div} \varphi=0$ because of [16, Lemma 2.44, Chapter 5]. Moreover, since $\tilde{S} \in L^{q^{\prime}}(Q)$, equation 5.10 implies that $\partial_{t} v \in L^{q^{\prime}}\left(0, T ; V_{q}(\Omega)^{\prime}\right)$ for all $T>0$. Therefore we can choose $\varphi=\left.v\right|_{Q_{T}}$ in 5.10 to obtain

$$
\frac{1}{2}\|v(T)\|_{2}^{2}+(\tilde{S}, D v)_{Q_{T}}=\frac{1}{2}\left\|v_{0}\right\|_{2}^{2},
$$

where we have used 4.8 and $(v \otimes v, \nabla v)_{Q_{T}}=0$ (cf. [16, Lemma 4.45, Chapter 5]). Moreover,

$$
\frac{1}{2}\left\|v_{k}(T)\right\|_{2}^{2}+\left(S\left(\chi_{k}, D v_{k}\right), D v_{k}\right)_{Q_{T}}=\frac{1}{2}\left\|v_{0}\right\|_{2}^{2}
$$

and therefore

$$
\begin{aligned}
\limsup _{k \rightarrow \infty}\left(S\left(\chi_{k}, D v_{k}\right), D v_{k}\right)_{Q_{T}} & =\frac{1}{2}\left\|v_{0}\right\|_{2}^{2}-\liminf _{k \rightarrow \infty} \frac{1}{2}\left\|v_{k}(T)\right\|_{2}^{2} \\
& \leqslant \frac{1}{2}\left\|v_{0}\right\|_{2}^{2}-\frac{1}{2}\|v(T)\|_{2}^{2}=(\tilde{S}, D v)_{Q_{T}} .
\end{aligned}
$$

Thus we are in a position to apply Theorem 2.6 with $A(x, s, \xi)=S(s, \xi), z_{k}=D v_{k}, y_{k}=\chi_{k}$ to conclude that for a suitable subsequence $\lim _{k \rightarrow \infty} S\left(\chi_{k}, D v_{k}\right)=\tilde{S}$ in measure. Since $T>0$ is arbitrary, this implies (5.9).

Proof of Theorem 1.9. For the case $\kappa=0$ the results obtained so far show that $(v, \chi)$ is a weak solution of 1.21 for $f=0$ together with $1.10-1.11$. The general case $f \in L^{q^{\prime}}\left(0, \infty ; V_{q}(\Omega)^{\prime}\right)$ can be proved in the same way with minor modifications.

REMARK 5.5 The condition $q \geqslant 2 d /(d+2)+1$ is only needed to estimate the convective term as in 5.11). For all other parts of the proof only $q>2 d /(d+2)$ is needed. Hence in the case of the Stokes equations, where the convective term $v \cdot \nabla v$ is neglected (cf. Remark 1.10), the condition $q>2 d /(d+2)$ is sufficient to prove existence of weak solutions. 


\subsection{Case with surface tension: properties of the interface}

It remains to consider the case with surface tension $\kappa>0$. For this let $\Omega_{k}^{+}(t)=X_{k, t}\left(\Omega_{0}^{+}\right)$, where $X_{k, t}=X_{\Psi_{k} v_{k}}(t)$ is the flow map associated to 2.14 - 2.15) with $v=\Psi_{k} v_{k}$ as described above. Moreover, let $\Gamma_{k}(t)=\partial \Omega_{k}^{+}(t)=X_{k, t}\left(\partial \Omega_{0}^{+}\right)$and let $\Gamma_{k}=\bigcup_{0 \leqslant t<\infty} \Gamma_{k}(t) \times\{t\}$.

First we will show that in the case $q>d$ or $d=2, \Gamma_{k} \cap Q_{T}$ is contained in the compact set $\overline{B_{R}(0)} \times[0, T]$ for $R=R(T)$ and arbitrary $T>0$. Then a suitable subsequence will converge in the Hausdorff distance.

Lemma 5.6 Let $d=2, \kappa>0$. Then $\Gamma_{k}(t) \subseteq \overline{B_{R}(0)}$ for all $t \in(0, T)$ and some $R=$ $R\left(T, E_{0}, \Omega_{0}^{+}\right)$.

Proof. Since $\mathcal{H}^{d-1}\left(\Gamma_{k}(t)\right) \leqslant \kappa^{-1} E_{0}$, obviously $\operatorname{diam}\left(\Omega_{k}^{+}(t)\right) \leqslant E_{0} / 2 \kappa$. Moreover, by the transport equation

$$
\int_{\Omega_{k}^{+}(t)} x \mathrm{~d} x=\int_{\Omega_{0}^{+}} x \mathrm{~d} x+\int_{0}^{t} \int_{\Omega_{k}^{+}(\tau)} v_{k} \cdot \mathbf{1} \mathrm{d} x \mathrm{~d} \tau,
$$

where $\mathbf{1}=(1, \ldots, 1)^{T}$, which implies

$$
\left|\int_{\Omega_{k}^{+}(t)} x \mathrm{~d} x\right| \leqslant C\left(\Omega_{0}^{+}\right)+t\left|\Omega_{0}^{+}\right|^{1 / 2} \sup _{0 \leqslant \tau \leqslant t}\|v(\tau)\|_{2} \leqslant C\left(T, E_{0}, \Omega_{0}^{+}\right)
$$

for all $0 \leqslant t \leqslant T$ since $\left|\Omega_{k}^{+}(\tau)\right|=\left|\Omega_{0}^{+}\right|$for all $\tau>0$. Therefore $\overline{\Omega_{k}^{+}(t)} \subseteq \overline{B_{R}(0)}$ for $0 \leqslant t \leqslant T$ with $R=C\left(T, E_{0}, \Omega_{0}^{+}\right)+E_{0} / 2 \kappa$.

In the case $q>d, v \in L^{q}\left(0, T ; C_{0}\left(\mathbb{R}^{d}\right)\right)$ since $V_{q}\left(\mathbb{R}^{d}\right) \cap L_{\sigma}^{2}\left(\mathbb{R}^{d}\right) \hookrightarrow C_{0}\left(\mathbb{R}^{d}\right)$ and we can prove that $\Gamma_{k}(t)$ are equi-Hölder continuous in the following sense:

LEMMA 5.7 Let $q>d$. Then

$$
d_{H}\left(\Gamma_{k}\left(t_{1}\right), \Gamma_{k}\left(t_{2}\right)\right) \leqslant C\left|t_{1}-t_{2}\right|^{1 / q^{\prime}}
$$

for all $0 \leqslant t_{1}, t_{2} \leqslant T, T>0$, where $C$ depends only on $E_{0}, q, T$. In particular, $\Gamma_{k}(t) \subseteq \overline{B_{R}(0)}$ for all $0 \leqslant t \leqslant T$ for some $R=R\left(T, E_{0}, \Omega_{0}^{+}\right)$.

Proof. By symmetry it suffices to show that $\Gamma_{k}\left(t_{1}\right) \subseteq\left(\Gamma_{k}\left(t_{2}\right)\right)_{\varepsilon}$ for $\varepsilon=C\left|t_{1}-t_{2}\right|^{1 / q^{\prime}}$. Let $x_{1} \in$ $\Gamma_{k}\left(t_{1}\right)$. Then by definition of $\Gamma_{k}(t)$ there is a curve $x(t)$ such that $x\left(t_{1}\right)=x_{1}$ and $x^{\prime}(t)=v_{k}(x(t), t)$ for $t>0$. Moreover, $x_{2}=x\left(t_{2}\right) \in \Gamma_{k}\left(t_{2}\right)$ and

$$
\left|x_{1}-x_{2}\right| \leqslant \int_{t_{1}}^{t_{2}}\left|v_{k}(x(t), t)\right| \mathrm{d} t \leqslant C\left(E_{0}, T, q\right)\left|t_{1}-t_{2}\right|^{1 / q^{\prime}}, \quad 0 \leqslant t_{1} \leqslant t_{2} \leqslant T .
$$

This proves the statement.

COROLlaRY 5.8 Let $\kappa>0$ and let $q>d$ or let $d=2$. Then there is a subsequence of $\Gamma_{k}, k \in \mathbb{N}$, (denoted again by $\Gamma_{k}, k \in \mathbb{N}$ ) and a closed set $\Gamma^{*} \subset \bar{Q}$ such that for every rational $T>0$,

$$
\Gamma_{k}^{*} \cap \bar{Q}_{T} \rightarrow \Gamma_{T}^{*} \quad \text { with respect to } d_{H} \text { as } k \rightarrow \infty
$$

for some compact set $\Gamma_{T}^{*} \subseteq \bar{Q}_{T}$ with $\Gamma_{T}^{*} \cap Q_{T}=\Gamma^{*} \cap Q_{T}$. 
Proof. By the previous two lemmas, $\Gamma_{k} \cap \bar{Q}_{T}$ is contained in a compact set $A_{T}$. Hence using the compactness of the metric space $\left(\mathcal{K}(A), d_{H}\right)$ for compact $A \subset \mathbb{R}^{N}$ one easily gets a subsequence of $\Gamma_{k}, k \in \mathbb{N}$, that converges in $\left(\mathcal{K}\left(A_{T}\right), d_{H}\right)$ for every rational $T>0$ to some compact set $\Gamma_{T}^{*}$. By the definition of $d_{H}$ one easily verifies that $\Gamma_{T_{1}}^{*} \cap Q_{T_{1}}=\Gamma_{T_{2}}^{*} \cap Q_{T_{1}}$ if $0<T_{1} \leqslant T_{2}$. From this the existence of $\Gamma^{*} \subseteq \bar{Q}$ is immediate.

In the case $q>d$, we even obtain:

Corollary 5.9 Let $q>d$. Then $\Gamma_{k}(t) \rightarrow \Gamma_{t}^{*}$ for all $t>0$ in the Hausdorff distance as $k \rightarrow \infty$, where $\Gamma_{t}^{*}=\left\{x \in \mathbb{R}^{d}:(x, t) \in \Gamma^{*}\right\}$.

Proof. First of all, for a fixed $t>0$ and a suitable subsequence, $\Gamma_{k_{j}}(t) \rightarrow \Gamma_{t}^{* *}$ in the Hausdorff distance as $j \rightarrow \infty$. We claim that $\Gamma_{t}^{* *}=\Gamma_{t}^{*}$. The inclusion $\Gamma_{t}^{* *} \subseteq \Gamma_{t}^{*}$ is obvious. Conversely, let $(x, t) \in \Gamma_{t}^{*}$. Then there is a sequence $\left(x_{k_{j}}, t_{k_{j}}\right) \in \Gamma_{k_{j}}$ such that $\lim _{j \rightarrow \infty}\left(x_{k_{j}}, t_{k_{j}}\right)=(x, t)$. But by Lemma 5.7 there are $y_{j} \in \Gamma_{k_{j}}(t)$ such that $\left|y_{j}-x_{k_{j}}\right| \leqslant C\left(E_{0}, T\right)\left|t-t_{k_{j}}\right|^{1 / q^{\prime}}$. Hence

$$
\left|x-y_{j}\right| \leqslant\left|x-x_{k_{j}}\right|+\left|x_{k_{j}}-y_{j}\right| \leqslant\left|x-x_{k_{j}}\right|+C\left(E_{0}\right)\left|t-t_{k_{j}}\right|^{1 / q^{\prime}},
$$

which shows that $\Gamma_{k_{j}}(t) \ni y_{j} \rightarrow x \in \Gamma_{t}^{*}$. Thus $\Gamma_{t}^{*} \subseteq \Gamma_{t}^{* *}$. Therefore $\Gamma_{t}^{*}=\Gamma_{t}^{* *}$ for any accumulation point $\Gamma_{t}^{* *}$ of $\Gamma_{k}(t)$ in the Hausdorff distance, which implies $\Gamma_{k}(t) \rightarrow \Gamma_{t}^{*}$ for all $t>0$ as $k \rightarrow \infty$.

The last corollary gives some compactness in time for the sequence of interfaces $\Gamma_{k}(t)$ if $q>d$ for $d=2,3$. But now there is a crucial difference between the cases $d=2$ and $d=3$. If $d=3$ and $t>0$ is fixed, then the boundedness of $\mathcal{H}^{d-1}\left(\Gamma_{k}(t)\right)$ does not imply that a limit of $\Gamma_{k}(t)$ in the Hausdorff distance has finite $\mathcal{H}^{d-1}$-measure. (It is easy to construct sequences of surfaces of fixed area with many "small fingers" that will converge to a set of positive Lebesgue measure.) This cannot happen in dimension two as the following lemma shows:

LEMmA 5.10 Let $\Gamma_{k} \subset \mathbb{R}^{2}, k \in \mathbb{N}$, be a sequence of compact Lipschitz curves and $\Gamma_{k} \rightarrow \Gamma^{*}$ in the Hausdorff distance for some compact set $\Gamma^{*} \subset \mathbb{R}^{2}$. Then $\mathcal{H}^{1}\left(\Gamma^{*}\right) \leqslant \liminf _{k \rightarrow \infty} \mathcal{H}^{1}\left(\Gamma_{k}\right)$.

Proof. Let $\delta>0$ and $q>1$ be fixed. Then there is an $N=N(\delta, q)$ such that $d_{H}\left(\Gamma_{k}, \Gamma^{*}\right) \leqslant$ $(1-1 / q) \delta / 2$ for all $k \geqslant N$. Moreover, for any $\varepsilon>0$ there is some $k_{\varepsilon} \geqslant N$ such that $L:=$ $\mathcal{H}^{1}\left(\Gamma_{k_{\varepsilon}}\right) \leqslant \liminf \operatorname{in}_{k \rightarrow \infty} \mathcal{H}^{1}\left(\Gamma_{k}\right)+\varepsilon$. Then there is a covering $\Gamma_{k_{\varepsilon}} \subseteq \bigcup_{j=1}^{M} \frac{{ }_{\varepsilon}}{B_{\delta / 2 q}\left(x_{j}\right)}$ such that $M \leqslant$ $(q / \delta) \mathcal{H}^{1}\left(\Gamma_{k_{\varepsilon}}\right)+1$. (Let $\gamma_{k}:[0, L] \rightarrow \mathbb{R}^{d}$ be a parameterization by arc-length and $t_{k}=k \cdot(\delta / q)$, $k=0, \ldots, M-1$, where $M=[(q / \delta) L]+1$, and $t_{M}=L$; then choose $x_{j}=\frac{1}{2}\left(\gamma_{k}\left(t_{j-1}\right)+\gamma_{k}\left(t_{j}\right)\right)$.) Therefore $\Gamma^{*} \subseteq \bigcup_{j=1}^{M} \overline{B_{\delta / 2}\left(x_{j}\right)}$ and

$$
\mathcal{H}_{\delta}^{1}\left(\Gamma^{*}\right) \leqslant \delta M \leqslant q \mathcal{H}^{1}\left(\Gamma_{k_{\varepsilon}}\right)+\delta \leqslant q\left(\liminf _{k \rightarrow \infty} \mathcal{H}^{1}\left(\Gamma_{k}\right)+\varepsilon\right)+\delta
$$

where

$$
\mathcal{H}_{\delta}^{1}(A)=\inf \left\{\sum_{j=1}^{\infty} 2 r_{j}: A \subseteq \bigcup_{j=1}^{\infty} B_{r_{j}}\left(x_{j}\right), 0<r_{j} \leqslant \delta\right\} .
$$

Since $q>1$ and $\varepsilon>0$ are arbitrary, $\mathcal{H}_{\delta}^{1}\left(\Gamma^{*}\right) \leqslant \liminf _{k \rightarrow \infty} \mathcal{H}^{1}\left(\Gamma_{k}\right)+\delta$ for every $\delta>0$, which proves the lemma.

Corollary 5.11 Let $q>d=2$ and $\kappa>0$. Then $\mathcal{H}^{1+q^{\prime}}\left(\Gamma^{*} \cap \bar{Q}_{T}\right)<\infty$ for all $T>0$. 
Proof. By Corollary 5.9, $\Gamma_{k}(t) \rightarrow \Gamma_{t}^{*}$ in the Hausdorff distance as $k \rightarrow \infty$. Moreover, by Lemma 5.10 $\mathcal{H}^{1}\left(\Gamma_{t}^{*}\right) \leqslant \liminf _{k \rightarrow \infty} \mathcal{H}^{1}\left(\Gamma_{k}(t)\right) \leqslant \kappa^{-1} E_{0}$ for all $t \geqslant 0$. Now choose $0=t_{0}<$ $t_{1}<\cdots<t_{N}=T, T>0$, with $\left|t_{j}-t_{j+1}\right| \leqslant \delta=(r / 3 C)^{q^{\prime}}$ for $r>0$ and $N \leqslant 2 T \delta^{-1}$, where $C$ is the same constant as in Lemma 5.7. Since the length of $\Gamma_{k}\left(t_{j}\right)$ is bounded by $\kappa^{-1} E_{0}$, there are balls $B_{r / 3}\left(x_{j, l}\right), l=1, \ldots, M_{j}, j=1, \ldots, N$, with $M_{j} \leqslant C\left(E_{0}\right) r^{-1}$ covering $\Gamma_{k}\left(t_{j}\right)$. Now choose $k \in \mathbb{N}$ so large that $d_{H}\left(\Gamma_{k}\left(t_{j}\right), \Gamma_{t_{j}}^{*}\right) \leqslant r / 3$ for $j=1, \ldots, N$. Using Lemma 5.7 and $\Gamma_{k}(t) \rightarrow \Gamma_{t}^{*}$, we conclude that

$$
d_{H}\left(\Gamma_{t_{1}}^{*}, \Gamma_{t_{2}}^{*}\right) \leqslant C\left|t_{1}-t_{2}\right|^{1 / q^{\prime}} .
$$

Then for $\left|t-t_{j}\right| \leqslant \delta$,

$$
\Gamma_{t}^{*} \subseteq\left(\Gamma_{t_{j}}^{*}\right)_{r / 3} \subseteq\left(\Gamma_{k}\left(t_{j}\right)\right)_{2 r / 3} \subseteq \bigcup_{l=1}^{M_{j}} B_{r}\left(x_{j, l}\right) .
$$

Thus

$$
\Gamma^{*} \cap \bar{Q}_{T} \subseteq \bigcup_{j=1}^{N} \bigcup_{l=1}^{M_{j}} B_{r}\left(x_{j, l}\right)
$$

where the number of balls on the right-hand side is bounded by $\mathrm{Cr}^{-1-q^{\prime}}$. Since $r>0$ was arbitrary, this implies that $\mathcal{H}^{1+q^{\prime}}\left(\Gamma^{*}\right) \leqslant C\left(E_{0}, q\right)$.

\subsection{Case with surface tension: end of proof}

Using Corollary 5.11 we obtain:

LEMMA 5.12 Let $\kappa>0$ and let $q>d=2$. Then

$$
S(\chi(x, t), D v(x, t))=\tilde{S}(x, t) \quad \text { for almost all }(x, t) \in Q .
$$

Proof. Because of Corollary 5.11, $\mathcal{H}^{3}\left(\Gamma^{*} \cap \bar{Q}_{T}\right)=0$ for all $T>0$. Hence $\overline{B_{R}(0)} \times[0, T]=$ $\bigcup_{j=1}^{\infty} Q_{j} \cup M$ with $M=\Gamma^{*} \cap \bar{Q}_{T}$ and $\mathcal{H}^{3}(M)=0$, where $Q_{j}=\left(a_{j}, b_{j}\right) \times B_{r_{j}}\left(x_{j}\right)$ and $\overline{Q_{j}} \cap M=\emptyset$. Now it is sufficient to prove that $(5.12)$ holds for all $\varphi$ with $\operatorname{supp} \varphi \subset Q_{j}$, which shall be arbitrary but fixed in the following. Then we choose $\eta \in C_{0}^{\infty}\left(Q_{j}\right)$ with $\eta \equiv 1$ on $\operatorname{supp} \varphi$. Because of the convergence of $\Gamma_{k}$ in Hausdorff distance, for every fixed $j \in \mathbb{N}$ we have $\left(\Gamma_{k}\right)_{\varepsilon_{k}} \cap Q_{j}=\emptyset$ for sufficiently large $k \in \mathbb{N}$. Hence $\chi_{\varepsilon_{k}}=l \in\{0,1\}$ is constant on $Q_{j}$ for suitably large $k$, and $w_{k}:=P_{L_{\sigma}^{2}\left(\mathbb{R}^{2}\right)}\left(\eta v_{k}\right) \in L^{\infty}\left(0, \infty ; L_{\sigma}^{2}\left(\mathbb{R}^{2}\right)\right) \cap L^{q}\left(0, \infty ; V_{q}\left(\mathbb{R}^{2}\right)\right)$ solves

$$
-\left(w_{k}, \partial_{t} u\right)_{Q}+\left(\left.w_{k}\right|_{t=0}, u(0)\right)_{\mathbb{R}^{2}}-\left(\Psi_{k} w_{k} \otimes w_{k}, \nabla u\right)_{Q}+\left(S\left(l, D w_{k}\right), D u\right)_{Q}=\left\langle f_{k}, u\right\rangle
$$

for all $u \in C_{(0)}^{\infty}\left([0, \infty) \times \mathbb{R}^{2}\right)^{2}$ with $\operatorname{div} u=0$, with a right-hand side $f_{k}$ satisfying

$$
f_{k} \rightarrow f \in L^{q^{\prime}}\left(0, \infty ; V_{q}\left(\mathbb{R}^{2}\right)^{\prime}\right) \quad \text { as } k \rightarrow \infty .
$$

Moreover, $w_{k} \rightarrow w$ in $L^{q}\left(0, \infty ; V_{q}\left(\mathbb{R}^{2}\right)\right)$ and weak-* in $L^{\infty}\left(0, \infty ; L_{\sigma}^{2}\left(\mathbb{R}^{2}\right)\right)$, and it can be shown by the same argument as in the case $\kappa=0$ (cf. Lemma 5.4) that $D w_{k} \rightarrow D w$ in measure. In particular this implies $\tilde{S}=S(l, D v)=S(\chi, D v)$ almost everywhere on $\operatorname{supp} \varphi$. Since $\varphi \in C_{0}^{\infty}(Q)$ with $\operatorname{supp} \varphi \subset Q_{j}$ and $Q_{j}$ have been arbitrary, (5.12) follows. 
Finally, we consider the sequence of oriented general varifolds $V_{k}(t), t \in[0, \infty)$, associated to $\Gamma_{k}(t)$, i.e.,

$$
\left\langle V_{k}(t), \varphi\right\rangle:=\int_{\mathbb{R}^{d}} \varphi\left(x, n_{k}(x)\right) \mathrm{d}\left|\nabla \chi_{k}(t)\right|, \quad \varphi \in C_{0}\left(\mathbb{R}^{d} \times \mathbb{S}^{d-1}\right),
$$

where $n_{k}=-\nabla \chi_{k} /\left|\nabla \chi_{k}\right|$, and we set

$$
\left\langle V_{k}, \varphi\right\rangle=\int_{0}^{\infty}\left\langle V_{k}(t), \varphi(t)\right\rangle \mathrm{d} t \quad \text { for all } \varphi \in L^{1}\left(0, \infty ; C_{0}\left(\mathbb{R}^{d} \times \mathbb{S}^{d-1}\right)\right)
$$

Hence for a suitable subsequence

$$
V_{k} \rightarrow^{*} V \quad \text { in } L^{\infty}\left(0, \infty ; H^{-s}\left(\mathbb{R}^{d} \times \mathbb{S}^{d-1}\right)\right), s>(2 d-1) / 2,
$$

for some $V \in L_{\omega}^{\infty}\left(0, \infty ; \mathcal{M}\left(\mathbb{R}^{d} \times \mathbb{S}^{d-1}\right)\right)$ since $V_{k} \in L_{\omega}^{\infty}\left(0, \infty ; \mathcal{M}\left(\mathbb{R}^{d} \times \mathbb{S}^{d-1}\right)\right)$ is uniformly bounded and $\mathcal{M}\left(\mathbb{R}^{d} \times \mathbb{S}^{d-1}\right) \hookrightarrow H^{-s}\left(\mathbb{R}^{d} \times \mathbb{S}^{d-1}\right)$. Then by choosing a test function of the form $\varphi(x, s, t)=s \cdot \psi(x, t), \psi \in C_{(0)}^{\infty}\left([0, \infty) \times \mathbb{R}^{d}\right)^{d}$, this implies

$$
\begin{aligned}
-\int_{0}^{\infty}\left\langle\nabla \chi_{k}(t), \psi(t)\right\rangle \mathrm{d} t & =\int_{0}^{\infty} \int_{\mathbb{R}^{d}} \int_{\mathbb{S}^{d-1}} s \cdot \psi(x, t) \mathrm{d} \delta_{n_{k}(x, t)} \mathrm{d}\left|\nabla \chi_{k}(t)\right| \mathrm{d} t \\
& \rightarrow-\int_{0}^{\infty}\langle\nabla \chi(t), \psi(t)\rangle \mathrm{d} t=\int_{\Omega} \int_{\mathbb{S}^{d-1}} s \cdot \psi(x, t) \mathrm{d} V_{x}(t) \mathrm{d}|V(t)| \mathrm{d} t,
\end{aligned}
$$

which shows 1.15 . Similarly, choosing $\varphi(x, s, t)=(I-s \otimes s): \nabla \psi(x, t)$ we conclude that

$$
-\int_{0}^{\infty}\left\langle H_{\chi_{k}(t)}, \psi(t)\right\rangle \mathrm{d} t=\int_{0}^{\infty}\left\langle\delta V_{k}(t), \psi(t)\right\rangle \mathrm{d} t \rightarrow \int_{0}^{\infty}\langle\delta V(t), \psi(t)\rangle \mathrm{d} t
$$

for all $\psi \in C_{(0)}^{\infty}\left([0, \infty) \times \mathbb{R}^{d}\right)^{d}$.

Moreover, by Theorem 2.1 there is some $\mu \in L_{\omega}^{\infty}\left(Q ; \operatorname{Prob}\left(\mathbb{R}_{\text {sym }}^{d \times d}\right)\right)$ such that 1.16 holds and

$$
S\left(l, D v_{k}\right) \rightarrow \int S(l, \lambda) \mathrm{d} \mu_{x, t}(\lambda) \quad \text { in } L^{q^{\prime}}(Q)
$$

for every $l \in[0,1]$. But this implies

$$
S\left(\chi(x, t), D v_{k}\right) \rightarrow \int S(\chi(x, t), \lambda) \mathrm{d} \mu_{x, t}(\lambda) \quad \text { in } L^{q^{\prime}}(Q) .
$$

Moreover, $\chi_{k} \rightarrow \chi$ in measure (for a suitable subsequence) and $S\left(\chi_{k}, D v_{k}\right)$ is uniformly bounded in $L^{q^{\prime}}(Q)$. Therefore

$$
\lim _{k \rightarrow \infty}\left(S\left(\chi_{k}, D v_{k}\right), D \varphi\right)_{Q}=\lim _{k \rightarrow \infty}\left(S\left(\chi, D v_{k}\right), D \varphi\right)_{Q}=\left(\int S(\chi, \lambda) \mathrm{d} \mu_{x, t}(\lambda), D \varphi\right)_{Q}
$$

for each $\varphi \in C_{0}^{\infty}(Q)^{d}$, which proves 1.14 . Hence the existence of measure-valued varifold solutions is proved.

It remains to prove the remaining properties stated in Theorem 1.6 The first statement follows from Lemmas 5.6 and 5.7. The second statement is proved by first proving that for a suitable subsequence $\left|V_{k}(t)\right| \rightarrow|V(t)|$ in $\mathcal{M}\left(\mathbb{R}^{2}\right)$ for almost all $t>0$ and then using an argument due to Plotnikov [20]: 
Lemma 5.13 Let $q>d$ and let $\kappa>0$. Then there is a subsequence (again denoted by $\left|V_{k}(t)\right|$ ) such that

$$
\left|V_{k}(t)\right| \rightarrow^{*}|V(t)| \quad \text { in } \mathcal{M}\left(\mathbb{R}^{d}\right)
$$

for almost all $t>0$.

Proof. First, we define a measure $E_{k}(t)$ by

$$
\left\langle E_{k}(t), \varphi\right\rangle:=\kappa\left\langle\left|V_{k}(t)\right|, \varphi\right\rangle+\frac{1}{2} \int_{\mathbb{R}^{d}}\left|v_{k}(x, t)\right|^{2} \varphi(x) \mathrm{d} x, \quad \varphi \in C_{0}\left(\mathbb{R}^{d}\right) .
$$

Note that $E_{k}(t)$ measures approximately the kinetic energy and "surface energy" at a given time $t>0$. We now show that $E_{k}(t)$ converges weak-* in measure almost everywhere (for a suitable subsequence).

By 2.8 we have

$$
\begin{aligned}
\frac{\mathrm{d}}{\mathrm{d} t}\left\langle\left|V_{k}(t)\right|, \varphi\right\rangle & =\frac{\mathrm{d}}{\mathrm{d} t} \int_{\Gamma_{k}(t)} \varphi(x, t) \mathrm{d} \mathcal{H}^{1}(x) \\
& =\left\langle\delta V_{k}(t), \varphi \Psi_{k} v_{k}(t)\right\rangle+\int_{\mathbb{R}^{d} \times \mathbb{S}^{d-1}} s \cdot \nabla \varphi(x) s \cdot \Psi_{k} v_{k}(x, t) \mathrm{d} V_{k}(t)(x, s) .
\end{aligned}
$$

Since $v_{k} \in L^{q}\left(0, T ; C_{0}\left(\mathbb{R}^{d}\right)\right), T>0$, is uniformly bounded, the last term in the equation above is uniformly bounded in $L^{q^{\prime}}\left(0, T ; C_{0}^{1}\left(\mathbb{R}^{d}\right)^{\prime}\right)$. Moreover,

$$
\left\langle\delta V_{k}(t), \varphi \Psi_{k} v_{k}\right\rangle=\left\langle\delta V_{k}(t), P_{\sigma}\left(\varphi \Psi_{k} v_{k}\right)\right\rangle+\left\langle\delta V_{k}(t),\left(I-P_{\sigma}\right)\left(\varphi \Psi_{k} v_{k}\right)\right\rangle,
$$

where $\left(I-P_{\sigma}\right)\left(\varphi \Psi_{k} v_{k}\right) \in L^{q}\left(0, T ; C_{0}^{1}\left(\mathbb{R}^{d}\right)\right), T>0$, and $\left\langle\delta V_{k}(\tau), \cdot\right\rangle \in L^{\infty}\left(0, \infty ; C_{0}^{1}\left(\mathbb{R}^{d}\right)^{\prime}\right)$ are uniformly bounded for every $\varphi \in C_{0}^{1}\left(\mathbb{R}^{d}\right)$. (Note that in the case $\Omega=\mathbb{R}^{d}$ the Helmholtz projection $P_{\sigma}$ can be represented using classical singular integral operators.) Therefore the second term in the equation above is also uniformly bounded in $L^{q^{\prime}}\left(0, T ; C_{0}^{1}\left(\mathbb{R}^{d}\right)^{\prime}\right)$. Furthermore,

$$
\left.\left\langle\delta V_{k}(t), P_{q}\left(\varphi\left(\Psi_{k} v_{k}\right)\right)\right\rangle=\left\langle\delta V_{k}(t), \Psi_{k} P_{q}\left(\varphi v_{k}\right)\right)\right\rangle-\left\langle\delta V_{k}(t), P_{q}\left[\Psi_{k}, \varphi\right] v_{k}\right\rangle
$$

where $[A, B]$ denotes the commutator of two operators. Note that $P_{\sigma}$ and $\Psi_{k}$ commute and that $P_{\sigma}$ is a bounded operator on $C^{\alpha}\left(\mathbb{R}^{d}\right) \cap L^{2}\left(\mathbb{R}^{d}\right)$, for all $\alpha \in(0,1)$. Moreover,

$$
\left\|\left[\Psi_{k}, \varphi\right] w\right\|_{C^{1, \alpha}\left(\mathbb{R}^{d}\right)} \leqslant C\|w\|_{C^{\alpha}\left(\mathbb{R}^{d}\right)}, \quad w \in C^{\alpha}\left(\mathbb{R}^{d}\right), 0<\alpha<1,
$$

uniformly in $k \in \mathbb{N}$. This implies that the second term in the equation above is uniformly bounded in $L^{q^{\prime}}\left(0, T ; C_{0}^{1}\left(\mathbb{R}^{d}\right)^{\prime}\right)$. On the other hand, by 4.1$)$,

$$
\begin{aligned}
-\kappa\left\langle\delta V_{k}(t), \Psi_{k} P_{\sigma}\left(\varphi v_{k}\right)\right\rangle= & -\kappa\left\langle H_{\Gamma_{k}(t)}, \Psi_{k} P_{\sigma}\left(\varphi v_{k}\right)\right\rangle \\
= & \frac{\mathrm{d}}{\mathrm{d} t} \frac{1}{2} \int_{\mathbb{R}^{d}}\left|v_{k}(x, t)\right|^{2} \varphi(x) \mathrm{d} x+\left\langle\left(1-P_{\sigma}\right)\left(\varphi v_{k}(t)\right), \partial_{t} v_{k}(t)\right\rangle \\
& +\left(\Psi_{k} v_{k} \otimes \psi_{k} * v_{k}, \nabla \psi_{k} * P_{\sigma}\left(\varphi v_{k}\right)\right)_{\mathbb{R}^{d}}+\left(S\left(\chi_{k}, D v_{k}\right), D P_{\sigma}\left(\varphi v_{k}\right)\right)_{\mathbb{R}^{d}},
\end{aligned}
$$

where the second term vanishes and the last two terms are again uniformly bounded in $L^{1}\left(0, T ; C_{0}^{1}\left(\mathbb{R}^{d}\right)^{\prime}\right)$. (Note that $v_{k} \otimes v_{k} \in L^{\infty}\left(0, T ; L^{1}\left(\mathbb{R}^{d}\right)\right) \cap L^{1}\left(0, T ; L^{\infty}\left(\mathbb{R}^{d}\right)\right) \hookrightarrow L^{1}\left(Q_{T}\right) \cap$ $L^{2}\left(Q_{T}\right)$ and $\nabla v_{k} \in L^{q}\left(Q_{T}\right)$ are uniformly bounded.) Summing up, we see that

$$
\frac{\mathrm{d}}{\mathrm{d} t}\left\langle E_{k}(t), \cdot\right\rangle \in L^{1}\left(0, T ; C_{0}^{1}\left(\mathbb{R}^{d}\right)^{\prime}\right)
$$


is uniformly bounded. Hence

$$
E_{k} \rightarrow \tilde{E} \quad \text { in } L^{p}\left(0, T ; H_{\mathrm{loc}}^{-s}\left(\mathbb{R}^{d}\right)\right) \text { if } s>2
$$

for every $1 \leqslant p<\infty$ by 2.1 and therefore $E_{k}(t) \rightarrow \tilde{E}(t)$ in $H_{\mathrm{loc}}^{-s}\left(\mathbb{R}^{d}\right)$ for almost all $t \in(0, T)$. On the other hand, $v_{k} \rightarrow v$ in $L^{q^{\prime}}\left(0, T ; L_{\text {loc }}^{2}\left(\mathbb{R}^{d}\right)\right)$ by Lemma 5.3 and therefore $v_{k}(t) \rightarrow v(t)$ in $L_{\text {loc }}^{2}\left(\mathbb{R}^{d}\right)$ for almost all $t \in(0, T)$ and for a suitable subsequence. Hence

$$
\left|V_{k}(t)\right| \rightarrow \mu(t) \quad \text { in } H_{\mathrm{loc}}^{-s}\left(\mathbb{R}^{d}\right)
$$

for almost all $t \in(0, T)$. But, since $C_{0}^{\infty}\left(\mathbb{R}^{d}\right)$ is dense in $C_{0}\left(\mathbb{R}^{d}\right)$ and $\left|V_{k}(t)\right|$ is uniformly bounded in $\mathcal{M}\left(\mathbb{R}^{d}\right)$, we conclude that

$$
\left|V_{k}(t)\right| \rightarrow^{*} \mu(t) \quad \text { in } \mathcal{M}\left(\mathbb{R}^{d}\right)
$$

for almost all $t \in(0, T)$. Finally, by 5.13 ,

$$
\left|V_{k}\right| \rightarrow^{*}|V| \quad \text { in } L^{\infty}\left(0, \infty ; H^{-s}\left(\mathbb{R}^{d}\right)\right) \text { for } s>(2 d-1) / 2
$$

and therefore $\mu=|V|$.

LEMma 5.14 Let $q>d=2$. Then $|V(t)|$ is supported on $\Gamma_{t}^{*}$ and $|V(t)| \geqslant \mathcal{H}^{1}\left\lfloor\Gamma_{t}^{*}\right.$ for almost all $t>0$.

Proof. First assume that $\Omega_{0}^{+}$is simply connected. Let $t>0$ be such that $\left|V_{k}(t)\right| \rightarrow^{*}|V(t)|$ in $\mathcal{M}\left(\mathbb{R}^{2}\right)$. Moreover, let $x_{k}:[0,1] \rightarrow \mathbb{R}^{2}$ be a parameterization of $\Gamma_{k, t}$ with respect to arclength times the total length $\mathcal{H}^{1}\left(\Gamma_{k, t}\right)$. Then $x_{k} \in C^{0,1}\left([0,1] ; \mathbb{R}^{2}\right)$ is uniformly bounded since $\Gamma_{k, t} \subseteq \overline{B_{R}(0)}$ for some $R>0$, and the Lipschitz constants of $x_{k}$ are $\mathcal{H}^{1}\left(\Gamma_{k, t}\right) \leqslant C\left(E_{0}\right)$. Hence for a suitable subsequence, $x_{k_{j}} \rightarrow x \in C^{0}\left([0,1] ; \mathbb{R}^{2}\right)$ for some $x \in C^{0,1}\left([0,1] ; \mathbb{R}^{2}\right)$ and $\mathcal{H}^{1}\left(\Gamma_{k_{j}, t}\right) \rightarrow l^{*}$. (Note that $\mathcal{H}^{1}\left(\Gamma_{k_{j}, t}\right)$ are bounded below since they enclose $\Omega_{k}^{+}(t)$ and $\left|\Omega_{k}^{+}(t)\right|=\left|\Omega_{0}^{+}\right|$.) Then

$$
\langle|V(t)|, \varphi\rangle=\lim _{j \rightarrow \infty} \mathcal{H}^{1}\left(\Gamma_{k_{j}, t}\right) \int_{0}^{1} \varphi\left(x_{k}(s)\right) \mathrm{d} s=l^{*} \int_{0}^{1} \varphi(x(s)) \mathrm{d} s
$$

for all $\varphi \in C_{0}\left(\mathbb{R}^{2}\right)$. Hence $\operatorname{supp}|V(t)|=x([0,1])$.

Now we prove that $\Gamma_{t}^{*}=x([0,1])$. Obviously, $x([0,1]) \subseteq \Gamma_{t}^{*}$. Conversely, if $x_{0} \in \Gamma_{t}^{*}$, then $x_{0}=\lim _{j \rightarrow \infty} x_{k_{j}}\left(s_{j}\right)$ for some $s_{j} \in[0,1]$. But then $s_{j} \rightarrow s_{0} \in[0,1]$ for a suitable subsequence again denoted by $s_{j}$. Hence $x_{0}=\lim _{j \rightarrow \infty} x_{k_{j}}\left(s_{j}\right)=x\left(s_{0}\right) \in x([0,1])$. This proves the first part of the lemma.

In order to prove $|V(t)| \geqslant \mathcal{H}^{1}\left\lfloor\Gamma^{*}\right.$ we use the fact that

$$
\langle|V(t)|, \varphi\rangle=l^{*} \int_{0}^{1} \varphi(x(s)) \mathrm{d} s \geqslant \int_{0}^{1} \varphi(x(s))\left|x^{\prime}(s)\right| \mathrm{d} s
$$

since $\left|x^{\prime}(s)\right| \leqslant l^{*}$ almost everywhere. Hence by the area formula

$$
|V(t)|(A) \geqslant \int_{0}^{1} \chi_{A}(x(s))\left|x^{\prime}(s)\right| \mathrm{d} s \geqslant \mathcal{H}^{1}\left\lfloor\Gamma_{t}^{*}(A)\right.
$$

for every open $A \subseteq \mathbb{R}^{2}$ (cf. e.g. [27]).

Finally, if $\Omega_{0}^{+}$is not simply connected, we apply the argument above to $N$-curves instead of one curve, where $N$ is the number of connected components of $\partial \Omega_{0}^{+}$. 


\section{A. Appendix: Rectifiability of the varifold}

One of the most challenging questions concerning measure-valued varifold solutions of the twophase flow with surface tension is whether there are solutions such that the unoriented general varifold $\widetilde{V}(t)$ associated to $V(t)$ via 2.7) is a $(d-1)$-rectifiable varifold for almost all $t>0$, i.e., $\widetilde{V}_{x}(t)=\delta_{P(x, t)}$ and

$$
\left\langle\widetilde{V}_{x}(t), \varphi\right\rangle=\int \varphi(x, P(x, t)) \theta_{t}(x) \mathrm{d} \mathcal{H}^{d-1}\left\lfloor M_{t}(x), \quad \varphi \in C_{0}\left(\Omega \times G_{d-1}\right),\right.
$$

for some countably $(d-1)$-rectifiable set $M_{t}$ and an $\mathcal{H}^{d-1}\left\lfloor M_{t}\right.$-measurable positive function $\theta_{t}$ (cf. [27]). In particular, the case that $\theta_{t}(x)$ is a positive integer for almost all $(x, t)$ would give a more satisfactory answer to the existence of measure-valued solutions.

As noted by Plotnikov [19], the major problem is that 11.14 gives only information on $\langle\delta V, \psi\rangle$ for $\psi \in C_{0}^{\infty}\left(Q ; \mathbb{R}^{d}\right)$ with $\operatorname{div} \psi=0$. But in order to apply techniques from geometric measure theory it is necessary to have a good estimate of $\langle\delta V(t), \psi\rangle$ for $\psi \in C_{0}^{\infty}\left(Q ; \mathbb{R}^{d}\right)$ with div $\psi \neq 0$ or at least for suitable gradients. The following result on regularity of measure-valued varifold solutions shows that, once $\langle\delta V, \psi\rangle$ can be estimated for all $\psi \in C_{0}^{\infty}\left(Q ; \mathbb{R}^{d+1}\right)$ in suitable norms and the $(d-1)$-density of $|V(t)|$ is bounded below, then $\widetilde{V}(t)$ is a $(d-1)$-rectifiable varifold. The result is based on a new rectifiability result for general varifolds due to Luckhaus [14].

TheOREM A.1 (Rectifiability) Let $(v, \chi, V, \mu)$ be a measure-valued solution as in Definition 1.2 and let $T>0$ and $q>2 d /(d+2)$. Assume that

$$
\limsup _{\rho \rightarrow 0} \rho^{-d+1}|V(t)|\left(B_{\rho}(x)\right) \geqslant \Theta_{t}>0
$$

for $|V(t)|$-almost all $x \in \mathbb{R}^{d}$ and almost all $t \in(0, T)$. If for some $s>1$,

$$
\langle\delta V, \cdot\rangle \in L^{1}\left(0, T ; W_{s, \mathrm{loc}}^{-1}\left(\mathbb{R}^{d}\right)\right),
$$

or if there is some $p \in L^{1}\left(0, T ; L_{\mathrm{loc}}^{s}\left(\mathbb{R}^{d}\right)\right)$ for some $s>1$ such that

$$
\begin{aligned}
\left(v, \partial_{t} \varphi\right)_{Q_{T}} & +\left(v_{0}, \varphi(0)\right)_{\mathbb{R}^{d}}-(v \otimes v, \nabla \varphi)_{Q_{T}} \\
& +\left(\int S(\chi, \lambda) \mathrm{d} \mu_{x, t}(\lambda), D \varphi\right)_{Q_{T}}-(p, \operatorname{div} \varphi)_{Q_{T}}=-\kappa \int_{0}^{T}\langle\delta V(t), \varphi(t)\rangle \mathrm{d} t
\end{aligned}
$$

for all $\varphi \in C_{(0)}^{\infty}\left([0, T) \times \mathbb{R}^{d} ; \mathbb{R}^{d}\right)$, then $\widetilde{V}(t)$ is a $(d-1)$-rectifiable varifold for almost all $t \in(0, T)$.

We note that, if $q>d=2$, then the measure-valued varifold solution of Theorem 1.6 satisfies

$$
\limsup _{\rho \rightarrow 0} \rho^{-1}|V(t)|\left(B_{\rho}(x)\right) \geqslant 1
$$

for $|V(t)|$-almost all $x$ and almost all $t>0$. Hence the lower bound of the $(d-1)$-density above is satisfied in this case.

The proof of Theorem A.1 is based on the following rectifiability theorem: 
THEOREM A.2 (Luckhaus [14]) Let $V$ be a (general) $(d-1)$-dimensional varifold on a domain $\Omega \subseteq \mathbb{R}^{d}$ whose first variation can be represented as

$$
\langle\delta V, \psi\rangle=\int(v \psi+A: \nabla \psi) \mathrm{d} \mu_{1}, \quad \psi \in C_{0}^{1}\left(\Omega ; \mathbb{R}^{d}\right)
$$

satisfying the estimate

$$
\begin{aligned}
\rho^{-d} \int_{B_{\rho}(x)}|A(y)| \mathrm{d} \mu_{1}(y)+\rho^{-(d-1)} \int_{B_{\rho}(x)}|v(y)| \mathrm{d} \mu_{1}(y) & \\
& \leqslant \partial_{\rho} F\left(\rho, \sup _{\rho<R<\operatorname{dist}(x, \partial \Omega)} R^{-(d-1)} \int_{B_{R}(x)} \mathrm{d} \mu_{2}\right)
\end{aligned}
$$

for all $B_{\rho}(x) \subseteq \Omega$ where $\mu_{1}, \mu_{2}$ are non-negative Radon measures on $\Omega$ and $F: \mathbb{R}_{+} \times \mathbb{R}_{+} \rightarrow$ $[0, \infty)$ satisfies

1. $F(0, L)=0, \partial_{\rho} F(\rho, L) \geqslant 0, \partial_{\rho}^{2} F(\rho, L) \leqslant 0$ for $\rho, L \geqslant 0$,

2. $\lim _{L \rightarrow \infty} L^{-1} g(L)=0$ where $g(L)=\inf \left\{R^{-d+1}+F(R, L): R>0\right\}$.

Moreover, assume that $\limsup _{\rho \rightarrow 0} \rho^{-d+1} \int_{B_{\rho}(x)} \mathrm{d}|V| \geqslant \theta>0$ for $|V|$-almost all $x \in \Omega$. Then $V$ is a $(d-1)$-rectifiable varifold.

REMARK A.3 We note that in the proof of Theorem A.2 the identity A2 is only needed if $\psi=\nabla \varphi$ is a gradient. For the convenience of the reader we repeat the first part of the proof of Theorem A.2 The monotonicity formula for

$$
u(\rho, x):=\rho^{-d+1} \int \phi\left(\frac{|x-y|}{\rho}\right) \mathrm{d}|V(t)|(y)
$$

is considered, where $\phi \in C^{\infty}([0, \infty))$ with $\phi^{\prime}(s) \leqslant 0, \phi(s)=1$ for $s \leqslant 1 / 2$, and $\phi(s)=0$ for $s \geqslant 1$. Then

$$
\begin{aligned}
\partial_{\rho} u(\rho, x)= & -(d-1) \rho^{-d} \int \phi\left(\frac{|x-y|}{\rho}\right) \mathrm{d}|V(t)|(y) \\
& -\rho^{-d} \int \phi^{\prime}\left(\frac{|x-y|}{\rho}\right) \frac{|x-y|}{\rho} \mathrm{d}|V(t)|(y) \\
= & \int \operatorname{Tr}\left(P \nabla_{y}\left[\frac{x-y}{\rho^{d}} \phi\left(\frac{|x-y|}{\rho}\right)\right]\right) \mathrm{d}|V(t)|(y) \\
& +\rho^{-d} \int\left[\left|P \frac{x-y}{|x-y|}\right|^{2}-1\right] \phi^{\prime}\left(\frac{|x-y|}{\rho}\right) \frac{|x-y|}{\rho} \mathrm{d}|V(t)|(y) \\
= & \left\langle\delta V(t),\left.\frac{x-y}{\rho^{d}} \phi\left(\frac{|x-y|}{\rho}\right)\right|^{2}\right. \\
& +\rho^{-d} \int\left|(I-P) \frac{x-y}{|x-y|}\right|^{2}\left|\phi^{\prime}\right|\left(\frac{|x-y|}{\rho}\right) \frac{|x-y|}{\rho} \mathrm{d}|V(t)|(y)
\end{aligned}
$$

where

$$
\frac{x-y}{\rho^{d}} \phi\left(\frac{|x-y|}{\rho}\right)=-\rho^{-d+2} \nabla_{y} \Phi\left(\frac{|x-y|}{\rho}\right) \quad \text { for } \Phi^{\prime}(s)=s^{-1} \phi(s)
$$


is a gradient field. Then the assumptions of the theorem are used to estimate $\left\langle\delta V(t), \frac{x-y}{\rho^{d}} \phi\left(\frac{|x-y|}{\rho}\right)\right\rangle$. In the rest of the proof $[\mathrm{A} 2]$ is not used.

Proof of Theorem A.1 First of all, since rectifiability is a local property, we replace $\mathbb{R}^{d}$ by $\Omega=$ $B_{R}(0)$ with $R>0$ arbitrary. Moreover, we can assume that $2 s \leqslant q^{*}$, where $1 / q^{*}=1 / q-1 / d<$ $1 / 2$.

First we consider the case where $\langle\delta V, \cdot\rangle \in L^{1}\left(0, T ; W_{s}^{-1}(\Omega)\right)$. Then there is some $A \in$ $L^{1}\left(0, T ; L^{S}(\Omega)\right)$ such that

$$
\langle\delta V, \psi\rangle=\int_{Q_{T}} A(x, t): \nabla \psi(x, t) \mathrm{d}(x, t) \quad \text { for all } \psi \in L^{\infty}\left(0, T ; W_{s^{\prime}, 0}^{1}(\Omega)\right),
$$

which easily follows from Hahn-Banach's theorem if we identify $W_{s^{\prime}, 0}^{1}(\Omega)$ with the closed subspace $\left\{\nabla \psi: \psi \in W_{s^{\prime}, 0}^{1}(\Omega)\right\} \subset L^{s^{\prime}}\left(\Omega ; \mathbb{R}^{d}\right)$. In order to apply Theorem A.2 we take for $\mu_{1}$ the $d$-dimensional Lebesgue measure and estimate

$$
\begin{aligned}
\rho^{-d} \int_{B_{\rho}(x)}|A(y)| \mathrm{d} y & \leqslant\left(\rho^{-d} \int_{B_{\rho}(x)} \mathrm{d} y\right)^{1 / s^{\prime}}\left(\rho^{-d} \int_{B_{\rho}(x)}|A(y)|^{s} \mathrm{~d} y\right)^{1 / s} \\
& =C \rho^{-1 / s}\left(\rho^{-d+1} \int_{B_{\rho}(x)}|A(y)|^{s} \mathrm{~d} y\right)^{1 / s} .
\end{aligned}
$$

Hence we can choose $F(\rho, L)=C \rho^{1 / s^{\prime}} L^{1 / s}$ for a suitable constant $C$ since $s>1$, and $\mu_{2}(M)=$ $\int_{M}|A(y)|^{s} \mathrm{~d} y$. It is easy to check that $F(\rho, L)$ satisfies condition 1 of the theorem. Moreover, choosing $\alpha=1 /(d s-1)$ we have

$$
g(L) \leqslant C\left(L^{\alpha(d-1)}+F\left(L^{-\alpha}, L\right)\right) \leqslant C^{\prime}\left(L^{\alpha(d-1)}+L^{-\alpha / s^{\prime}+1 / s}\right)=C^{\prime} L^{(d-1) /(d s-1)} .
$$

where $(d-1) /(d s-1)<1$ since $s>1$. Hence $\lim _{L \rightarrow \infty} L^{-1} g(L)=0$.

In the second case we first use A1 for gradients $\varphi(x, t)=\phi(t) \nabla \psi(x)$ for $\psi \in C_{0}^{\infty}(\Omega)$, $\phi \in C_{0}^{\infty}(0, T)$, which yields

$$
\begin{aligned}
\left|\kappa \int_{0}^{T}\langle\delta V(t), \nabla \psi\rangle \phi(t) \mathrm{d} t\right| \leqslant & C\left(\|v\|_{L^{1}\left(0, T ; L^{2 s}(\Omega)\right)}^{2}\|\phi\|_{L^{\infty}(0, T)}\left\|\nabla^{2} \psi\right\|_{L^{s^{\prime}}(\Omega)}\right. \\
& +\left\|\int S(\chi, \lambda) \mathrm{d} \mu_{x, t}\right\|_{L^{q^{\prime}}\left(Q_{T}\right)}\|\phi\|_{L^{\infty}(0, T)}\left\|\nabla^{2} \psi\right\|_{L^{q}(\Omega)} \\
& \left.+\|p\|_{L^{1}\left(0, T ; L^{s}(\Omega)\right)}\|\phi\|_{L^{\infty}(0, T)}\left\|\nabla^{2} \psi\right\|_{L^{s^{\prime}}(\Omega)}\right) \\
\leqslant & C(T)\left(E_{0}+\|p\|_{L^{1}\left(0, T ; L^{s}(\Omega)\right)}\right)\|\phi\|_{L^{\infty}(0, T)}\left\|\nabla^{2} \psi\right\|_{L^{s^{\prime}}(\Omega)} .
\end{aligned}
$$

Hence

$$
\langle\delta V, \cdot\rangle \in L^{1}\left(0, T ;\left(G_{s^{\prime}}^{1}(\Omega)\right)^{\prime}\right)
$$

where $G_{s^{\prime}}^{1}(\Omega)=\left\{\nabla \varphi \in W_{s^{\prime}}^{1}(\Omega): \varphi \in L^{s^{\prime}}(\Omega)\right\} \subset W_{s^{\prime}}^{1}(\Omega)$. In particular, $\langle\delta V(t), \cdot\rangle \in\left(G_{s^{\prime}}^{1}(\Omega)\right)^{\prime}$ for almost all $t \in(0, T)$ with $s>1$. Now we can apply the arguments of the first part since by Remark A.3 the identity (A2) is only needed for gradients. 


\section{Acknowledgements}

Part of the research leading to this article was done during a stay at the Department of Mathematics, Hokkaido University in Sapporo and was supported by the 21th century COE for mathematics. The author expresses his gratitude to the people at the Hokkaido University, in particular, to Professor Yoshikazu Giga, for their hospitality, for the financial support of the stay and for many interesting discussions. Moreover, special thanks are due to Stephan Luckhaus for an unpublished version of [14] and several stimulating discussions. Finally, the author is grateful to the referees for their careful reading of the manuscript and suggestions to improve the presentation of this article.

\section{REFERENCES}

1. Abels, H. The initial value problem for the Navier-Stokes equations with a free surface in $L^{q}$-Sobolev spaces. Adv. Differential Equations 10 (2005), 45-64. Zbl pre05056142 MR 2106120

2. Ambrosio, L., Fusco, N., \& Pallara, D. Functions of Bounded Variation and Free Discontinuity Problems. Oxford Math. Monogr., Clarendon Press, Oxford (2000). Zbl 0957.49001 MR 1857292

3. Beale, J. T. The initial value problem for the Navier-Stokes equations with a free surface. Comm. Pure Appl. Math. 34 (1981), 359-392. Zbl 0464.76028 MR 0611750

4. BeAle, J. T. Large-time regularity of viscous surface waves. Arch. Ration. Mech. Anal. 84 (1984), 307352. Zbl 0545.76029 MR 0721189

5. Bergh, J., \& LÖfström, J. Interpolation Spaces. Springer, Berlin (1976). Zbl 0344.46071 MR 0482275

6. Bronsard, L., Garcke, H., \& Stoth, B. A multi-phase Mullins-Sekerka system: matched asymptotic expansions and an implicit time discretisation for the geometric evolution problem. Proc. Roy. Soc. Edinburgh Sect. A 128 (1998), 481-506. Zbl 0924.35199 MR 1632810

7. Diamond, P., \& Kloeden, P. Metric Spaces of Fuzzy Sets. World Sci., River Edge, NJ (1994). Zbl 0873.54019 MR 1337027

8. Diestel, J., \& Uhl, J. J., Jr. Vector Measures. Amer. Math. Soc., Providence, RI (1977). Zbl 0369.46039 MR 0453964

9. DiPernA, R. J., \& LiONS, P.-L. Ordinary differential equations, transport theory and Sobolev spaces. Invent. Math. 98 (1989), 511-547. Zbl 0696.34049 MR 1022305

10. Giga, Y., \& TAKAhashi, S. On global weak solutions of the nonstationary two phase Stokes flow. SIAM J. Math. Anal. 25 (1994), 876-893. Zbl 0806.35137 MR 1271315

11. GomeZ, N., \& ZolÉSIO, J.-P. Existence of free-boundary for a two non-Newtonian fluids problem. Shape Optimization and Optimal Design (Cambridge, 1999), Lecture Notes in Pure and Appl. Math. 216, Dekker, New York (2001), 289-300. Zbl 0994.76006 MR 1816849

12. JosePh, D. D., \& RenARd, Y. Y. Fundamentals of Two-Fluid Dynamics. Part I: Mathematical Theory and Applications. Interdiscip. Appl. Math. 3, Springer, New York (1993). Zbl 0784.76002 MR 1200237

13. Lions, J.-L. Quelques méthodes de résolution des problèmes aux limites non linéaires. Dunod (1969). Zbl 0189.40603 MR 0259693

14. LuCKhaus, S. Uniform rectifiability from mean curvature bounds. Recent Advances in Elliptic and Parabolic Problems, World Sci. (2005), 197-201. MR 2172575

15. Luckhaus, S., \& Sturzenhecker, T. Implicit time discretization for the mean curvature flow equation. Calc. Var. Partial Differential Equations 3 (1995), 253-271. Zbl 0821.35003 MR 1386964

16. MÁlek, J., NeČAs, J., RokytA, M., \& RŮŽIČKA, M. Weak and Measure-Valued Solutions to Evolutionary PDEs. Appl. Math. Math. Comput. 13, Chapman \& Hall, London (1996). Zbl 0851.35002 MR 1409366 
17. Nouri, A., \& Poupaud, F. An existence theorem for the multifluid Navier-Stokes problem. J. Differential Equations 122 (1995), 71-88. Zbl $0842.35079 \mid$ MR 1356130

18. Nouri, A., Poupaud, F., \& Demay, Y. An existence theorem for the multi-fluid Stokes problem. Quart. Appl. Math. 55 (1997), 421-435. Zbl 0882.35091 MR 1466141

19. Plotnikov, P. I. On a class of curves that arises in a problem with a free boundary for Stokes flows. Sibirsk. Mat. Zh. 36 (1995), 619-627 (in Russian). Zbl 0861.76017 MR 1404886

20. Plotnikov, P. I. Generalized solutions to a free boundary problem of motion of a non-Newtonian fluid. Siberian Math. J. 34 (1993), 704-716. Zbl 0814.76007|| MR 1248797

21. Plotnikov, P. I. Compressible Stokes flow driven by capillarity on a free surface. Navier-Stokes Equations and Related Nonlinear Problems (Palanga, 1997), H. Amann et al. (eds.), VSP, Utrecht (1998), 217-238. Zbl 0948.35101 MR 1690711

22. RoubíčEK, T. A generalization of the Lions-Temam compact imbedding theorem. Časopis Pěst. Mat. 115 (1990), 338-342. Zbl 0755.46013 MR 1090857

23. Salvi, R. On the existence of free surface problem for viscous incompressible flow. Topics in Mathematical Fluid Mechanics (Capo Miseno, 2000), P. G. Galdi et al. (eds.), Quad. Mat. 10, Aracne, Rome (2002), 247-275. Zbl 1050.35089 MR 2051777

24. Shibata, Y., \& Shimizu, S. $L_{p}-L_{q}$ maximal regularity and viscous incompressible flows with free surface. Proc. Japan Acad. Ser. A Math. Sci. 81 (2005), 151-155. MR 2189671

25. Simader, C. G., \& Sohr, H. A new approach to the Helmholtz decomposition and the Neumann problem in $L_{q}$-spaces for bounded and exterior domains. Mathematical Problems Relating to the NavierStokes Equation, Ser. Adv. Math. Appl. Sci. 11, World Sci., Singapore (1992), 1-35. Zbl 0791.35096 MR 1190728

26. Simon, J. Compact sets in the space $L^{p}(0, T ; B)$. Ann. Mat. Pura Appl. (4) 146 (1987), 65-96. Zbl 0629.46031 MR 0916688

27. Simon, L. Lectures on Geometric Measure Theory. Proc. Centre Math. Anal. Austral. Nat. Univ. 3, Centre Math. Anal., Austral. Nat. Univ., Canberra (1983). Z Zbl 0546.49019 MR 0756417

28. Sohr, H. The Navier-Stokes Equations. Birkhäuser (2001). Zbl 0983.35004 MR 1928881

29. Solonnikov, V. A. Estimates of the solution of a certain initial-boundary value problem for a linear nonstationary system of Navier-Stokes equations. Zap. Nauchn. Sem. Leningrad. Otdel. Mat. Inst. Steklov. (LOMI) 59 (1976), 178-254, 257 (in Russian). Zbl 0357.76026 MR 0460931

30. Solonnikov, V. A. On the transient motion of an isolated volume of viscous incompressible fluid. Math. USSR-Izv. 31 (1988), 381-405. Zbl 0850.76180 MR 0925094

31. Soner, H. M. Convergence of the phase-field equations to the Mullins-Sekerka problem with kinetic undercooling. Arch. Ration. Mech. Anal. 131 (1995), 139-197. Zbl 0829.73010 MR 1346368

32. ŚWIERCZEWSKA, A. A dynamical approach to large eddy simulation of turbulent flows: existence of weak solutions. Math. Methods Appl. Sci. 29 (2006), 99-121. Zbl 1080.76035 MR 2185636

33. TANI, A., \& TANAKA, N. Large-time existence of surface waves in incompressible viscous fluids with or without surface tension. Arch. Ration. Mech. Anal. 130 (1995), 303-314. Zbl 0844.76025 MR 1346360

34. TRIEBEL, H. Interpolation Theory, Function Spaces, Differential Operators. North-Holland, Amsterdam (1978). Zbl 0387.46033 MR 0503903

35. WAgner, A. On the Bernoulli free-boundary problem. Differential Integral Equations 14 (2001), 51-58. Zbl 1022.35050 MR 1797931

36. ZeIDler, E. Nonlinear Functional Analysis and its Applications. II/B. Springer, New York (1990). Zbl 0684.47029 MR 1033498 